\title{
Kawasaki Dynamics with Two Types of Particles: Stable/Metastable Configurations and Communication Heights
}

\author{
F. den Hollander • F.R. Nardi • A. Troiani
}

Received: 27 May 2011 / Accepted: 24 September 2011 / Published online: 13 October 2011

(C) The Author(s) 2011. This article is published with open access at Springerlink.com

\begin{abstract}
This is the second in a series of three papers in which we study a two-dimensional lattice gas consisting of two types of particles subject to Kawasaki dynamics at low temperature in a large finite box with an open boundary. Each pair of particles occupying neighboring sites has a negative binding energy provided their types are different, while each particle has a positive activation energy that depends on its type. There is no binding energy between particles of the same type. At the boundary of the box particles are created and annihilated in a way that represents the presence of an infinite gas reservoir. We start the dynamics from the empty box and are interested in the transition time to the full box. This transition is triggered by a critical droplet appearing somewhere in the box.

In the first paper we identified the parameter range for which the system is metastable, showed that the first entrance distribution on the set of critical droplets is uniform, computed the expected transition time up to and including a multiplicative factor of order one, and proved that the nucleation time divided by its expectation is exponentially distributed, all in the limit of low temperature. These results were proved under three hypotheses, and involve three model-dependent quantities: the energy, the shape and the number of critical droplets. In the second paper we prove the first and the second hypothesis and identify the energy of critical droplets. In the third paper we settle the rest.

Both the second and the third paper deal with understanding the geometric properties of subcritical, critical and supercritical droplets, which are crucial in determining the metastable behavior of the system, as explained in the first paper. The geometry turns out to be considerably more complex than for Kawasaki dynamics with one type of particle, for which an extensive literature exists. The main motivation behind our work is to understand metastability of multi-type particle systems.
\end{abstract}

F. den Hollander · A. Troiani ( $\bowtie)$

Mathematical Institute, Leiden University, P.O. Box 9512, 2300 RA Leiden, The Netherlands

e-mail: atroiani@math.leidenuniv.nl

F. den Hollander · F.R. Nardi

EURANDOM, P.O. Box 513, 5600 MB Eindhoven, The Netherlands

F.R. Nardi

Technische Universiteit Eindhoven, P.O. Box 513, 5600 MB Eindhoven, The Netherlands 
Keywords Lattice gas · Multi-type particle systems · Kawasaki dynamics · Metastability · Critical configurations $\cdot$ Polyominoes $\cdot$ Discrete isoperimetric inequalities

\section{Introduction}

Section 1.1 defines the model, Sect. 1.2 introduces basic notation, Sect. 1.3 states the main theorems, while Sect. 1.4 discusses the main theorems and provides further perspectives.

\subsection{Lattice Gas Subject to Kawasaki Dynamics}

Let $\Lambda \subset \mathbb{Z}^{2}$ be a large box centered at the origin (later it will be convenient to choose $\Lambda$ rhombus-shaped). Let $(|\cdot|$ denotes the Euclidean norm)

$$
\begin{aligned}
& \partial^{-} \Lambda=\{x \in \Lambda: \exists y \notin \Lambda:|y-x|=1\}, \\
& \partial^{+} \Lambda=\{x \notin \Lambda: \exists y \in \Lambda:|y-x|=1\},
\end{aligned}
$$

be the internal, respectively, external boundary of $\Lambda$, and put $\Lambda^{-}=\Lambda \backslash \partial^{-} \Lambda$ and $\Lambda^{+}=\Lambda \cup$ $\partial^{+} \Lambda$. With each site $x \in \Lambda$ we associate a variable $\eta(x) \in\{0,1,2\}$ indicating the absence of a particle or the presence of a particle of type 1 or type 2 , respectively. A configuration $\eta=\{\eta(x): x \in \Lambda\}$ is an element of $\mathcal{X}=\{0,1,2\}^{\Lambda}$. To each configuration $\eta$ we associate an energy given by the Hamiltonian

$$
H=-U \sum_{(x, y) \in \Lambda^{*,-}} 1_{\{\eta(x) \eta(y)=2\}}+\Delta_{1} \sum_{x \in \Lambda} 1_{\{\eta(x)=1\}}+\Delta_{2} \sum_{x \in \Lambda} 1_{\{\eta(x)=2\}},
$$

where $\Lambda^{*--}=\left\{(x, y): x, y \in \Lambda^{-},|x-y|=1\right\}$ is the set of non-oriented bonds inside $\Lambda^{-}$, $-U<0$ is the binding energy between neighboring particles of different types inside $\Lambda^{-}$, and $\Delta_{1}>0$ and $\Delta_{2}>0$ are the activation energies of particles of type 1 , respectively, 2 inside $\Lambda$. Without loss of generality we will assume that

$$
\Delta_{1} \leq \Delta_{2}
$$

The Gibbs measure associated with $H$ is

$$
\mu_{\beta}(\eta)=\frac{1}{Z_{\beta}} e^{-\beta H(\eta)}, \quad \eta \in \mathcal{X}
$$

where $\beta \in(0, \infty)$ is the inverse temperature and $Z_{\beta}$ is the normalizing partition sum.

Kawasaki dynamics is the continuous-time Markov process, $\left(\eta_{t}\right)_{t \geq 0}$ with state space $\mathcal{X}$ whose transition rates are

$$
c_{\beta}\left(\eta, \eta^{\prime}\right)=e^{-\beta\left[H\left(\eta^{\prime}\right)-H(\eta)\right]_{+}}, \quad \eta, \eta^{\prime} \in \mathcal{X}, \eta \neq \eta^{\prime}, \eta \leftrightarrow \eta^{\prime},
$$

where $\eta \leftrightarrow \eta^{\prime}$ means that $\eta^{\prime}$ can be obtained from $\eta$ by one of the following moves:

- interchanging 0 and 1 or 0 and 2 between two neighboring sites in $\Lambda$ ("hopping of particles in $\Lambda$ "),

- changing 0 to 1 or 0 to 2 in $\partial^{-} \Lambda$ ("creation of particles in $\partial^{-} \Lambda^{\prime}$ ), 
- changing 1 to 0 or 2 to 0 in $\partial^{-} \Lambda$

("annihilation of particles in $\partial^{-} \Lambda^{\prime}$ ),

and $c_{\beta}\left(\eta, \eta^{\prime}\right)=0$ otherwise. Note that this dynamics preserves particles in $\Lambda^{-}$, but allows particles to be created and annihilated in $\partial^{-} \Lambda$. Think of the latter as describing particles entering and exiting $\Lambda$ along non-oriented bonds between $\partial^{+} \Lambda$ and $\partial^{-} \Lambda$ (the rates of these moves are associated with the bonds rather than with the sites). The pairs $\left(\eta, \eta^{\prime}\right)$ with $\eta \leftrightarrow \eta^{\prime}$ are called communicating configurations, the transitions between them are called allowed moves. Note that particles in $\partial^{-} \Lambda$ do not interact: the interaction only works in $\Lambda^{-}$ (see (1.2)). Also note that the Gibbs measure is the reversible equilibrium of the Kawasaki dynamics:

$$
\mu_{\beta}(\eta) c_{\beta}\left(\eta, \eta^{\prime}\right)=\mu_{\beta}\left(\eta^{\prime}\right) c_{\beta}\left(\eta^{\prime}, \eta\right) \quad \forall \eta, \eta^{\prime} \in \mathcal{X} .
$$

The dynamics defined by (1.2) and (1.5) models the behavior inside $\Lambda$ of a lattice gas in $\mathbb{Z}^{2}$, consisting of two types of particles subject to random hopping with hard-core repulsion and with binding between different neighboring types. We may think of $\mathbb{Z}^{2} \backslash \Lambda$ as an infinite reservoir that keeps the particle densities fixed at $\rho_{1}=e^{-\beta \Delta_{1}}$ and $\rho_{2}=e^{-\beta \Delta_{2}}$. In the above model this reservoir is replaced by an open boundary $\partial^{-} \Lambda$, where particles are created and annihilated at a rate that matches these densities. Thus, the dynamics is a finite-state Markov process, ergodic and reversible with respect to the Gibbs measure $\mu_{\beta}$ in (1.4).

Note that there is no binding energy between neighboring particles of the same type (including such an interaction would make the model much more complicated). Consequently, our dynamics has an "anti-ferromagnetic flavor", and does not reduce to Kawasaki dynamics for one type of particle when $\Delta_{1}=\Delta_{2}$. Also note that our dynamics does not allow swaps between particles, i.e., interchanging 1 and 1 or 2 and 2 or 1 and 2 between two neighboring sites in $\Lambda$. (The first two would not effect the dynamics, but the third would; for Kawasaki dynamics with one type of particle swaps between 1 and 1 have no effect.)

See Sects. 1.3-1.4 for a further discussion on the choice of the parameters $U, \Delta_{1}, \Delta_{2}$.

\subsection{Notation}

To state our main theorems in Sect. 1.3, we need some notation.

\section{Definition 1.1}

(a) $\square$ is the configuration where $\Lambda$ is empty.

(b) $\boxplus$ is the set consisting of the two configurations where $\Lambda$ is filled with the largest possible checkerboard droplet such that all particles of type 2 are surrounded by particles of type 1 .

(c) $\omega: \eta \rightarrow \eta^{\prime}$ is any path of allowed moves from $\eta \in \mathcal{X}$ to $\eta^{\prime} \in \mathcal{X}$.

(d) $\Phi\left(\eta, \eta^{\prime}\right)$ is the communication height between $\eta, \eta^{\prime} \in \mathcal{X}$ defined by

$$
\Phi\left(\eta, \eta^{\prime}\right)=\min _{\omega: \eta \rightarrow \eta^{\prime}} \max _{\xi \in \omega} H(\xi)
$$

and $\Phi(A, B)$ is its extension to non-empty sets $A, B \subset \mathcal{X}$ defined by

$$
\Phi(A, B)=\min _{\eta \in A, \eta^{\prime} \in B} \Phi\left(\eta, \eta^{\prime}\right) .
$$


(e) $V_{\eta}$ is the stability level of $\eta \in \mathcal{X}$ defined by

$$
V_{\eta}=\Phi\left(\eta, \mathcal{I}_{\eta}\right)-H(\eta)
$$

where $\mathcal{I}_{\eta}=\{\xi \in \mathcal{X}: H(\xi)<H(\eta)\}$ is the set of configurations with energy lower than $\eta$.

(f) $\mathcal{X}_{\text {stab }}=\left\{\eta \in \mathcal{X}: H(\eta)=\min _{\xi \in \mathcal{X}} H(\xi)\right\}$ is the set of stable configurations, i.e., the set of configurations with minimal energy.

(g) $\mathcal{X}_{\text {meta }}=\left\{\eta \in \mathcal{X}: V_{\eta}=\max _{\xi \in \mathcal{X} \backslash \mathcal{X}_{\text {stab }}} V_{\xi}\right\}$ is the set of metastable configurations, i.e., the set of non-stable configurations with maximal stability level.

(h) $\Gamma=V_{\eta}$ for $\eta \in \mathcal{X}_{\text {meta }}$ (note that $\eta \mapsto V_{\eta}$ is constant on $\mathcal{X}_{\text {meta }}$ ), $\Gamma^{\star}=\Phi(\square, \boxplus)-H(\square)$ (note that $H(\square)=0$ ).

In [3] we were interested in the transition of the Kawasaki dynamics from $\square$ to $\boxplus$ in the limit as $\beta \rightarrow \infty$. This transition, which is viewed as a crossover from a "gas phase" to a "liquid phase", is triggered by the appearance of a critical droplet somewhere in $\Lambda$. The critical droplets form a subset of the set of configurations realizing the energetic minimax of the paths of the Kawasaki dynamics from $\square$ to $\boxplus$, which all have energy $\Gamma^{\star}$ because $H(\square)=0$.

In [3] we showed that the first entrance distribution on the set of critical droplets is uniform, computed the expected transition time up to and including a multiplicative factor of order one, and proved that the nucleation time divided by its expectation is exponentially distributed, all in the limit as $\beta \rightarrow \infty$. These results, which are typical for metastable behavior, were proved under three hypotheses:

(H1) $\mathcal{X}_{\text {stab }}=\boxplus$.

(H2) There exists a $V^{\star}<\Gamma^{\star}$ such that $V_{\eta} \leq V^{\star}$ for all $\eta \in \mathcal{X} \backslash\{\square$, $\boxplus\}$.

(H3) A hypothesis about the shape of the configurations in and near the essential gate for the transition from $\square$ to $\boxplus$ (for details see [3]).

As shown in [3], (H1-H3) are the geometric input that is needed to derive the metastability theorems in [3] with the help of the potential-theoretic approach to metastability outlined in Bovier [2]. In the present paper we prove $(\mathrm{H} 1-\mathrm{H} 2)$ and identify the energy $\Gamma^{\star}$ of critical droplets. In [4] we prove (H3) and identify the configurations that form the critical droplets.

Lemma $1.2(\mathrm{H} 1-\mathrm{H} 2)$ imply that $V_{\square}=\Gamma^{\star}$. Consequently, by Definition $1.1(\mathrm{~g}-\mathrm{h}), \Gamma=\Gamma^{\star}$ and $\mathcal{X}_{\text {meta }}=\square$.

Proof By Definition 1.1(e-h) and (H1), $\boxplus \in \mathcal{I}_{\square}$, which implies that $V_{\square} \leq \Gamma^{\star}$. We show that (H2) implies $V_{\square}=\Gamma^{\star}$. The proof is by contradiction. Suppose that $V_{\square}<\Gamma^{\star}$. Then, by Definition 1.1(h), there exists a $\eta_{0} \in \mathcal{I}_{\square} \backslash \boxplus$ such that $\Phi\left(\square, \eta_{0}\right)-H(\square)<\Gamma^{\star}$. But (H2), together with the finiteness of $\mathcal{X}$, implies that there exist an $m \in \mathbb{N}$ and a sequence $\eta_{1}, \ldots, \eta_{m} \in \mathcal{X}$ with $\eta_{m}=\boxplus$ such that $\eta_{i+1} \in \mathcal{I}_{\eta_{i}}$ and $\Phi\left(\eta_{i}, \eta_{i+1}\right) \leq H\left(\eta_{i}\right)+V^{\star}$ for $i=0, \ldots, m-1$. Therefore

$$
\Phi\left(\eta_{0}, \boxplus\right) \leq \max _{i=0, \ldots, m-1} \Phi\left(\eta_{i}, \eta_{i+1}\right) \leq \max _{i=0, \ldots, m-1}\left[H\left(\eta_{i}\right)+V^{\star}\right]=H\left(\eta_{0}\right)+V^{\star}<H(\square)+\Gamma^{\star},
$$

where in the first inequality we use that $\Phi(\eta, \sigma) \leq \max \{\Phi(\eta, \xi), \Phi(\xi, \sigma)\}$ for all $\eta, \sigma, \xi \in \mathcal{X}$, and in the last inequality that $\eta_{0} \in \mathcal{I}_{\square}$ and $V^{\star}<\Gamma^{\star}$. It follows that

$$
\Phi(\square, \boxplus)-H(\square) \leq \max \left\{\Phi\left(\square, \eta_{0}\right)-H(\square), \Phi\left(\eta_{0}, \boxplus\right)-H(\square)\right\}<\Gamma^{\star},
$$


Fig. 1 Proper metastable region

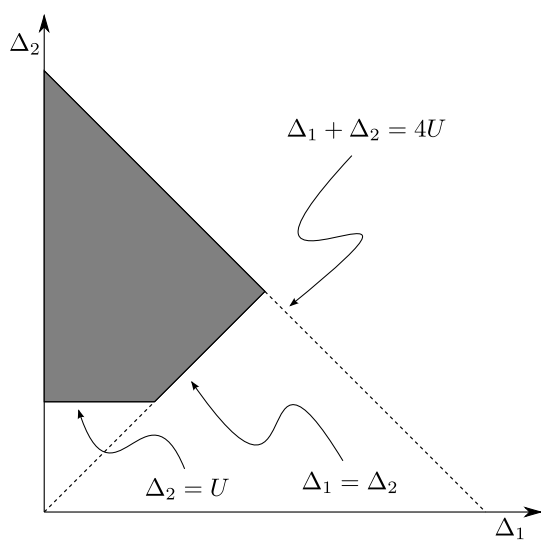

which contradicts Definition 1.1(h).

The claim that $\Gamma=\Gamma^{\star}$ follows from Definition $1.1(\mathrm{~g}-\mathrm{h})$. To see why $\mathcal{X}_{\text {meta }}=\square$, suppose that there exists a configuration $\eta_{m} \in \mathcal{X}_{\text {meta }} \backslash \square$. Then, by Definition 1.1(h), $V_{\eta_{m}}=V_{\square}=\Gamma^{\star}$, which contradicts $(\mathrm{H} 2)$.

Hypotheses $(\mathrm{H} 1-\mathrm{H} 2)$ imply that $\left(\mathcal{X}_{\text {meta }}, \mathcal{X}_{\text {stab }}\right)=(\square, \boxplus)$, and that the highest energy barrier between any two configurations in $\mathcal{X}$ is the one separating $\square$ and $\boxplus$, i.e., ( $\square$, $\boxplus$ ) is the unique metastable pair. Hypothesis (H3) is needed only to find the asymptotics of the prefactor of the expected transition time in the limit as $\Lambda \rightarrow \mathbb{Z}^{2}$. The main theorems in [3] involve three model-dependent quantities: the energy, the shape and the number of critical droplets.

\subsection{Main Theorems}

In [3] it was shown that $0<\Delta_{1}+\Delta_{2}<4 U$ is the metastable region, i.e., the region of parameters for which $\square$ is a local minimum but not a global minimum of $H$. Moreover, it was argued that within this region the subregion where $\Delta_{1}, \Delta_{2}<U$ is of no interest because the critical droplet consists of two free particles, one of type 1 and one of type 2 . Therefore the proper metastable region is

$$
0<\Delta_{1} \leq \Delta_{2}, \quad \Delta_{1}+\Delta_{2}<4 U, \quad \Delta_{2} \geq U,
$$

as indicated in Fig. 1.

In this present paper, the analysis will be carried out for the subregion of the proper metastable region where

$$
\Delta_{1}<U, \quad \Delta_{2}-\Delta_{1}>2 U, \quad \Delta_{1}+\Delta_{2}<4 U,
$$

as indicated in Fig. 2. Note: The second and third restriction imply the first restriction. Nevertheless, we write all three because each plays an important role in the sequel.

The following three theorems are the main result of the present paper and are valid subject to (1.13). We write $\lceil\cdot\rceil$ to denote the upper integer part.

Theorem 1.3 $\mathcal{X}_{\text {stab }}=\boxplus$ 
Fig. 2 Subregion of the proper metastable region given by (1.13)
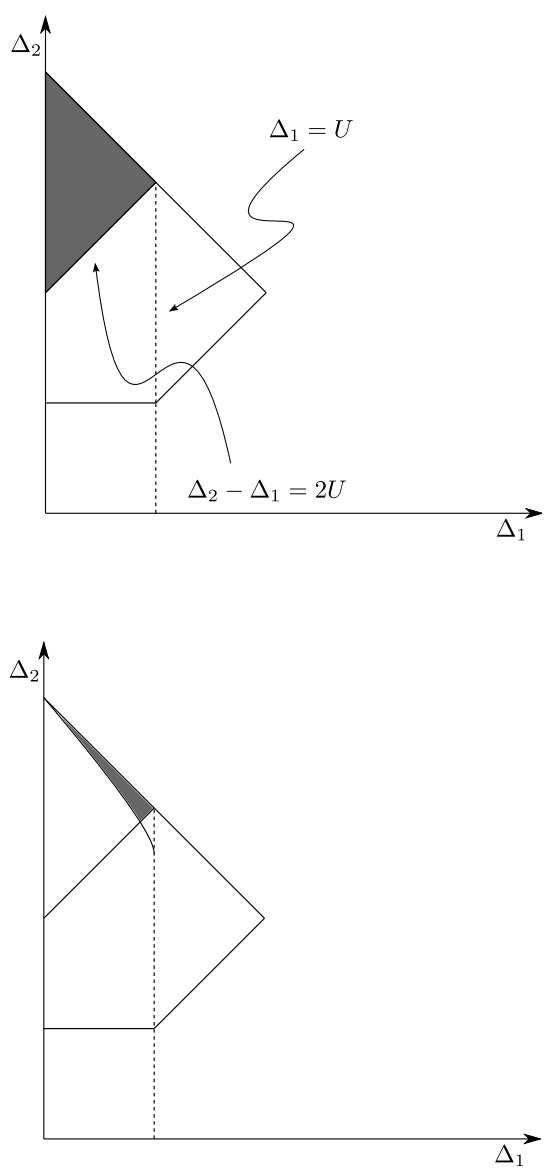

Fig. 3 The parameter region where $\Gamma^{\star}>10 U-\Delta_{1}$ contains the shaded region

Theorem 1.4 There exists $a V^{\star} \leq 10 U-\Delta_{1}$ such that $V_{\eta} \leq V^{\star}$ for all $\eta \in \mathcal{X} \backslash\{\square, \boxplus\}$. Consequently, if $\Gamma^{\star}>10 U-\Delta_{1}$, then $(\mathrm{H} 1-\mathrm{H} 2)$ hold and, by Lemma $1.2, \mathcal{X}_{\text {meta }}=\square$ and $\Gamma=\Gamma^{\star}$.

Theorem 1.5 $\Gamma^{\star}=-\left[\ell^{\star}\left(\ell^{\star}-1\right)+1\right]\left(4 U-\Delta_{1}-\Delta_{2}\right)+\left(2 \ell^{\star}+1\right) \Delta_{1}+\Delta_{2}$ with

$$
\ell^{\star}=\left\lceil\frac{\Delta_{1}}{4 U-\Delta_{1}-\Delta_{2}}\right\rceil \in \mathbb{N} .
$$

Theorem 1.3 settles hypothesis (H1) in [3], Theorem 1.4 settles hypothesis (H2) in [3] when $\Gamma^{\star}>10 U-\Delta_{1}$, while Theorem 1.5 identifies $\Gamma^{\star}$, which is the energy of the critical droplets.

As soon as $V^{\star}<\Gamma^{\star}$, the energy landscape does not contain wells deeper than those surrounding $\square$ and $\boxplus$. Theorems 1.3 and 1.4 imply that this occurs at least when $\Gamma^{\star}>$ $10 U-\Delta_{1}$, while Theorem 1.5 identifies $\Gamma^{\star}$ and allows us to exhibit a further subregion of (1.13) where the latter inequality is satisfied. This further subregion contains the shaded region in Fig. 3. 
Fig. 4 A critical droplet. Light-shaded squares are particles of type 1, dark-shaded squares are particles of type 2 . The particles of type 2 form an $\ell^{\star} \times\left(\ell^{\star}-1\right)$ quasi-square with a protuberance attached to one of its longest sides, and are all surrounded by particles of type 1 . In addition, there is a free particle of type 2. As soon as this free particle attaches itself "properly" to a particle of type 1 the dynamics is "over the hill" (see [3], Sect. 2.3, item 3)

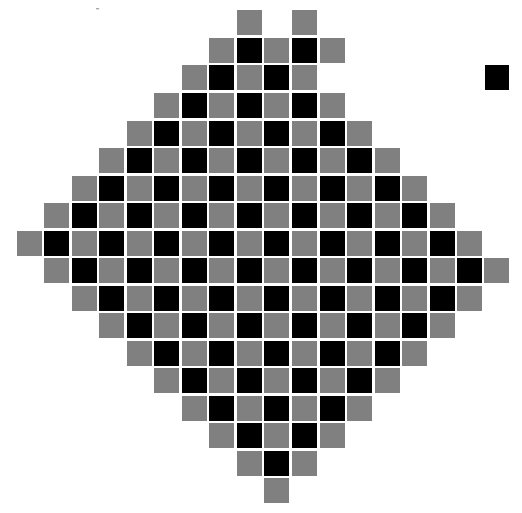

\subsection{Discussion}

1 In Sect. 4 we will see that the critical droplets for the crossover from $\square$ to $\boxplus$ consist of a rhombus-shaped checkerboard with a protuberance plus a free particle, as indicated in Fig. 4 . The fact that the free particle is of type 2 is due to the fact that $\Delta_{2}>\Delta_{1}$. A more detailed description will be given in [4].

\section{Abbreviate}

$$
\varepsilon=4 U-\Delta_{1}-\Delta_{2}
$$

and write $\ell^{\star}=\left(\Delta_{1} / \varepsilon\right)+\iota$ with $\iota \in[0,1)$. Then an easy computation shows that $\Gamma^{\star}=$ $\left(\Delta_{1}\right)^{2} / \varepsilon+\Delta_{1}+4 U+\varepsilon \iota(1-\iota)$. From this we see that

$$
\ell^{\star} \sim \Delta_{1} / \varepsilon, \quad \Gamma^{\star} \sim\left(\Delta_{1}\right)^{2} / \varepsilon, \quad \varepsilon \downarrow 0 .
$$

The limit $\varepsilon \downarrow 0$ corresponds to the weakly supersaturated regime, where the lattice gas wants to condensate but the energetic threshold to do so is high (because the critical droplet is large). From the viewpoint of metastability this regime is the most interesting. The shaded region in Fig. 3 captures this regime for all $0<\Delta_{1}<U$. This region contains the set of parameters where $\left(\Delta_{1}\right)^{2} / \varepsilon+\Delta_{1}+4 U>10 U-\Delta_{1}$, i.e., $\varepsilon / U<\left(\Delta_{1} / U\right)^{2} /\left[6-2\left(\Delta_{1} / U\right)\right]$.

3 The simplifying features of (1.13) over (1.12) are the following: $\Delta_{1}<U$ implies that each time a particle of type 1 enters $\Lambda$ and attaches itself to a particle of type 2 in a droplet the energy goes down, while $\Delta_{2}-\Delta_{1}>2 U$ implies that no particle of type 2 sits on the boundary of a droplet that has minimal energy given the number of particles of type 2 in the droplet. In [3] we conjectured that the metastability results presented there actually hold throughout the region given by (1.12), even though the critical droplets will be different when $\Delta_{1} \geq U$.

As will become clear in Sect. 3, the constraint $\Delta_{1}<U$ has the effect that in all configurations that are local minima of $H$ all particles on the boundary of a droplet are of type 1 . It will turn out that such configurations consist of a single rhombus-shaped checkerboard droplet. We expect that as $\Delta_{1}$ increases from $U$ to $2 U$ there is a gradual transition from a rhombus-shaped checkerboard critical droplet to a square-shaped checkerboard critical droplet. This is one of the reasons why it is difficult to go beyond (1.13). 
4 What makes Theorem 1.4 hard to prove is that the estimate on $V_{\eta}$ has to be uniform in $\eta \notin\{\square, \boxplus\}$. In configurations containing several droplets and/or droplets close to $\partial^{-} \Lambda$ there may be a lack of free space making the motion of particles inside $\Lambda$ difficult. The mechanisms developed in Sect. 5 allow us to realize an energy reduction to a configuration that lies on a suitable reference path for the nucleation within an energy barrier $10 U-\Delta_{1}$ also in the absence of free space around each droplet.

We will see in Sect. 5 that for droplets sufficiently far away from other droplets and from $\partial^{-} \Lambda$ a reduction within an energy barrier $\leq 4 U+\Delta_{1}$ is possible. Thus, if we would be able to control the configurations that fail to have this property, then we would have $V^{\star} \leq$ $4 U+\Delta_{1}$ and, consequently, would have $\mathcal{X}_{\text {meta }}=\square$ and $\Gamma=\Gamma^{\star}$ throughout the subregion given by (1.13) because $\Gamma^{\star}>4 U+\Delta_{1}$.

Another way of phrasing the last observation is the following. We view the "liquid phase" as the configuration filling the entire box $\Lambda$. If, instead, we would let the liquid phase correspond to the set of configurations filling most of $\Lambda$ but staying away from $\partial^{-} \Lambda$, then the metastability results derived in [3] would apply throughout the subregion given by (1.13).

5 Theorems 1.3 and 1.5 can actually be proved without the restriction $\Delta_{2}-\Delta_{1}>2 U$. However, removal of this restriction makes the task of showing that in droplets with minimal energy all particles of type 2 are surrounded by particles of type 1 more involved than what is done in Sect. 3. We omit this extension, since the restriction $\Delta_{2}-\Delta_{1}>2 U$ is needed for Theorem 1.4 anyway.

6 In [3] we describe four classes of models that have a flavor similar to our model of Kawasaki dynamics with two types of particles: (1) Glauber dynamics of spins taking values $\{-1,0,+1\}$ (Blume-Capel model); (2) Glauber dynamics of Ising spins with an anisotropic interaction or in a staggered magnetic field; (3) Kawasaki dynamics of one type of particle with an anisotropic interaction; (4) probabilistic cellular automata. In each of these models the geometry of the energy landscape is highly complex, like in our model of Kawasaki dynamics with two types of particles, and considerable work is needed to arrive at a full description of the metastable behavior.

Outline Section 2 contains preparations. Theorems 1.3-1.5 are proved in Sects. 3-5, respectively. The proofs are purely combinatorial, and are rather involved due to the presence of two types of particles rather than one. Sections 3-4 deal with statics and Sect. 5 with dynamics. Section 5 is technically the hardest and takes up about half of the paper. More detailed outlines are given at the beginning of each section.

\section{Coordinates, Definitions and Polyominoes}

Section 2.1 introduces two coordinate systems that are used to describe the particle configurations: standard and dual. Section 2.2 lists the main geometric definitions that are needed in the rest of the paper. Section 2.3 proves a lemma about polyominoes (finite unions of unit squares) and Sect. 2.4 a lemma about 2-tiled clusters (checkerboard configurations where all particles of type 2 are surrounded by particles of type 1). These lemmas are needed in Sect. 3 to identify the droplets of minimal energy given the number of particles of type 2 in $\Lambda$. 
Fig. 5 A configuration represented in: (a) standard coordinates; (b) dual coordinates. Light-shaded squares are particles of type 1, dark-shaded squares are particles of type 2 . In dual coordinates, particles of type 2 are represented by larger squares than particles of type 1 to exhibit the "tiled structure" of the configuration

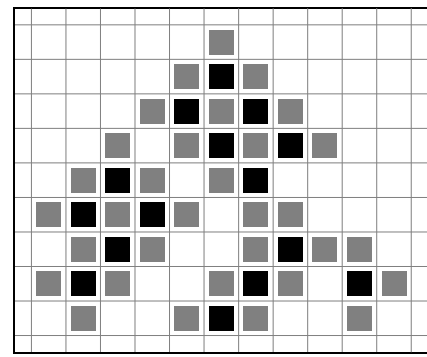

(a)

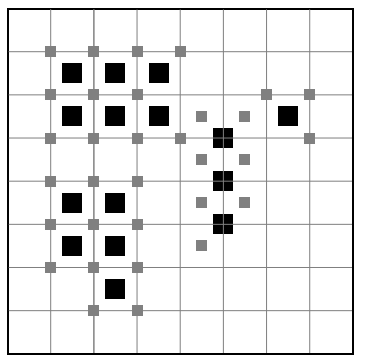

(b)

\subsection{Coordinates}

1 A site $i \in \Lambda$ is identified by its standard coordinates $x(i)=\left(x_{1}(i), x_{2}(i)\right)$, and is called odd when $x_{1}(i)+x_{2}(i)$ is odd and even when $x_{1}(i)+x_{2}(i)$ is even. The standard coordinates of a particle $p$ in $\Lambda$ are denoted by $x(p)=\left(x_{1}(p), x_{2}(p)\right)$. The parity of a particle $p$ is defined as $x_{1}(p)+x_{2}(p)+\eta(x(p))$ modulo 2 , and $p$ is said to be odd when the parity is 1 and even when the parity is 0 .

2 A site $i \in \Lambda$ is also identified by its dual coordinates

$$
u_{1}(i)=\frac{x_{1}(i)-x_{2}(i)}{2}, \quad u_{2}(i)=\frac{x_{1}(i)+x_{2}(i)}{2} .
$$

Two sites $i$ and $j$ are said to be adjacent, written $i \sim j$, when $\left|x_{1}(i)-x_{1}(j)\right|+\mid x_{2}(i)-$ $x_{2}(j) \mid=1$ or, equivalently, $\left|u_{1}(i)-u_{1}(j)\right|=\left|u_{2}(i)-u_{2}(j)\right|=\frac{1}{2}$ (see Fig. 5).

3 For convenience, we take $\Lambda$ to be the $\left(L+\frac{3}{2}\right) \times\left(L+\frac{3}{2}\right)$ dual square with bottom-left corner at site with dual coordinates $\left(-\frac{L+1}{2},-\frac{L+1}{2}\right)$ for some $L \in \mathbb{N}$ with $L>2 \ell^{\star}$ (to allow for $H(\boxplus)<H(\square)$; see Sect. 3.1). Particles interact only inside $\Lambda^{-}$, which is a $\left(L+\frac{1}{2}\right) \times$ $\left(L+\frac{1}{2}\right)$ dual square. This dual square, a rhombus in standard coordinates, is convenient because the local minima of $H$ are rhombus-shaped as well (see Sect. 3).

\subsection{Definitions}

1 A site $i \in \Lambda$ is said to be lattice-connecting in the configuration $\eta$ if there exists a lattice path $\lambda$ from $i$ to $\partial^{-} \Lambda$ such that $\eta(j)=0$ for all $j \in \lambda$ with $j \neq i$. We say that a particle $p$ is lattice-connecting if $x(p)$ is a lattice-connecting site.

2 Two particles in $\eta$ at sites $i$ and $j$ are called connected if $i \sim j$ and $\eta(i) \eta(j)=2$. If two particles $p_{1}$ and $p_{2}$ are connected, then we say that there is an active bond $b$ between them. The bond $b$ is said to be incident to $p_{1}$ and $p_{2}$. A particle $p$ is said to be saturated if it is connected to four other particles, i.e., there are four active bonds incident to $p$. The support of the configuration $\eta$, i.e., the union of the unit squares centered at the occupied sites of $\eta$, is denoted by $\operatorname{supp}(\eta)$. For a configuration $\eta, n_{1}(\eta)$ and $n_{2}(\eta)$ denote the number of particles of type 1 and 2 in $\eta$, and $B(\eta)$ denotes the number of active bonds. The energy of $\eta$ equals $H(\eta)=\Delta_{1} n_{1}(\eta)+\Delta_{2} n_{2}(\eta)-U B(\eta)$. 


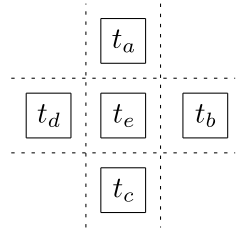

(a)

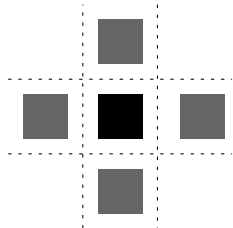

(b)

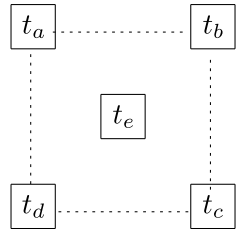

(c)

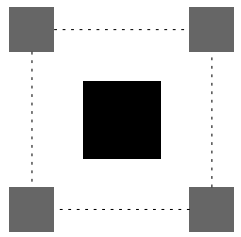

(d)

Fig. 6 Tiles: (a) standard representation of the labels of a tile; (b) standard representation of a 2-tile; (c) dual representation of the labels of a tile; (d) dual representation of a 2-tile

3 Let $G(\eta)$ be the graph associated with $\eta$, i.e., $G(\eta)=(V(\eta), E(\eta))$, where $V(\eta)$ is the set of sites $i \in \Lambda$ such that $\eta(i) \neq 0$, and $E(\eta)$ is the set of the pairs $\{i, j\}, i, j \in V(\eta)$, such that the particles at sites $i$ and $j$ are connected. A configuration $\eta^{\prime}$ is called a subconfiguration of $\eta$, written $\eta^{\prime} \prec \eta$, if $\eta^{\prime}(i)=\eta(i)$ for all $i \in \Lambda$ such that $\eta^{\prime}(i)>0$. A subconfiguration $c \prec \eta$ is a cluster if the graph $G(c)$ is a maximal connected component of $G(\eta)$. The set of non-saturated particles in $c$ is called the boundary of $c$, and is denoted by $\partial c$. Clearly, all particles in the same cluster have the same parity. Therefore the concept of parity extends from particles to clusters.

4 For a site $i \in \Lambda$, the tile centered at $i$, denoted by $\mathrm{t}(i)$, is the set of five sites consisting of $i$ and the four sites adjacent to $i$. If $i$ is an even site, then the tile is said to be even, otherwise the tile is said to be odd. The five sites of a tile are labeled $a, b, c, d, e$ as in Fig. 6. The sites labeled $a, b, c, d$ are called junction sites. If a particle $p$ sits at site $i$, then $\mathrm{t}(i)$ is also denoted by $\mathrm{t}(p)$ and is called the tile associated with $p$. In standard coordinates, a tile is a square of size $\sqrt{2}$. In dual coordinates, it is a unit square.

5 A tile whose central site is occupied by a particle of type 2 and whose junction sites are occupied by particles of type 1 is called a 2-tile (see Fig. 6). Two 2-tiles are said to be adjacent if their particles of type 2 have dual distance 1 . A horizontal (vertical) 12-bar is a maximal sequence of adjacent 2-tiles all having the same horizontal (vertical) coordinate. If the sequence has length 1 , then the 12-bar is called a 2-tiled protuberance. A cluster containing at least one particle of type 2 such that all particles of type 2 are saturated is said to be 2-tiled. A 2-tiled configuration is a configuration consisting of 2-tiled clusters only.

6 The tile support of a configuration $\eta$ is defined as

$$
[\eta]=\bigcup_{p \in \Phi_{2}(\eta)} \mathrm{t}(p)
$$

where $\varpi_{2}(\eta)$ is the set of particles of type 2 in $\eta$. Obviously, $[\eta]$ is the union of the tile supports of the clusters making up $\eta$. For a standard cluster $c$ the dual perimeter, denoted by $P(c)$, is the length of the Euclidean boundary of its tile support $[c]$ (which includes an inner boundary when $c$ contains holes). The dual perimeter $P(\eta)$ of a 2-tiled configuration $\eta$ is the sum of the dual perimeters of the clusters making up $\eta$.

$7 \mathcal{V}_{\star, n_{2}}$ is the set of configurations such that in $\left(\Lambda^{-}\right)^{-}$the number of particles of type 2 is $n_{2} . \mathcal{V}_{\star, n_{2}}^{4 n_{2}}$ is the set of configurations such that in $\left(\Lambda^{-}\right)^{-}$the number of particles of type 2 is $n_{2}$, the number of active bonds is $4 n_{2}$, and there are no non-interacting particles of type 1 . 
Fig. 7 Corners of polyominoes:

(a) one convex corner; (b) one concave corner; (c) two concave corners. Shaded mean occupied by a unit square

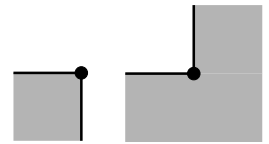

(a) (b)

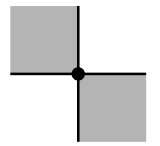

(c)

In other words, $\mathcal{V}_{\star, n_{2}}^{4 n_{2}}$ is the set of 2-tiled configurations with $n_{2}$ particles of type 2. A configuration $\eta$ is called standard if $\eta \in \mathcal{V}_{\star, n_{2}}^{4 n_{2}}$, and its tile support is a standard polyomino in dual coordinates (see Definition 2.1 below for the definition of a standard polyomino).

8 A unit hole is an empty site such that all four of its neighbors are occupied by particles of the same type (either all of type 1 or all of type 2 ). An empty site with three neighboring sites occupied by a particle of type 1 is called a good dual corner. In the dual representation a good dual corner is a concave corner (see Fig. 7).

\subsection{A Lemma on Polyominoes}

The tile support of a collection of clusters $c$ can be represented by polyominoes, i.e., finite unions of unit squares, with each polyomino representing a cluster on the dual lattice. The following notation is used:

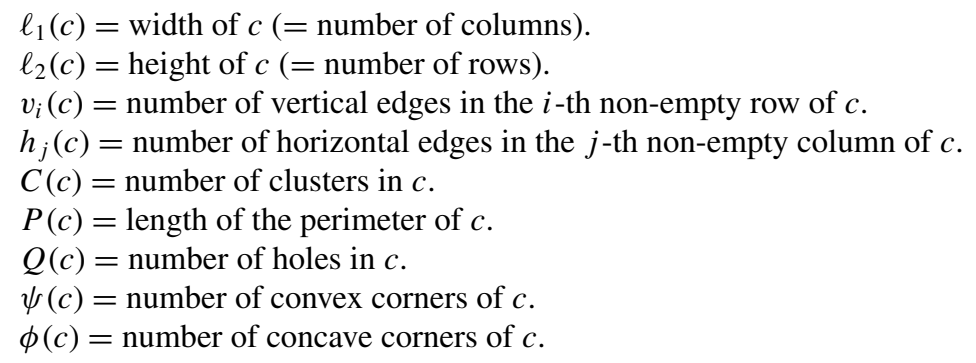

Note that $\psi(c)=\sum_{i=1}^{N(c)} \psi(i)$ and $\phi(c)=\sum_{i=1}^{N(c)} \phi(i)$, where $N(c)$ is the number of vertices along the perimeter of the polyomino representing $c$. If two edges $e_{1}$ and $e_{2}$ are incident to vertex $i$ at a right angle with a unit square inside and no unit squares outside, then $\psi(i)=1$ and $\phi(i)=0$ (Fig. 7(a)). On the other hand, if there is no unit square inside and three unit squares outside, then $\psi(i)=0$ and $\phi(i)=1$ (Fig. 7(b)). If four edges $e_{1}, e_{2}, e_{3}, e_{4}$ are incident to vertex $i$, with two unit squares in opposite angles, then $\psi(i)=0$ and $\phi(i)=2$ (Fig. 7(c)).

Definition 2.1 (Alonso and Cerf [1].) A polyomino is called monotone if its perimeter is equal to the perimeter of its circumscribing rectangle. A polyomino whose support is a quasi-square (i.e., a rectangle whose side lengths differ by at most one), with possibly a bar attached to one of its longest sides, is called a standard polyomino.

In the sequel, a key role will be played by the quantity

$$
\mathcal{T}(c)=2 P(c)+[\psi(c)-\phi(c)] .
$$

The geometric meaning of $\mathcal{T}(c)$ will be discussed at the beginning of the proof of Lemma 2.3. Note that

$$
\psi(c)-\phi(c)=4[C(c)-Q(c)]
$$


Fig. 8 Effect of vertical and horizontal projection

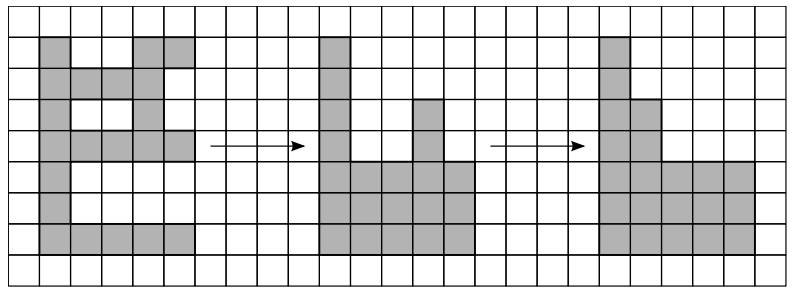

\section{Lemma 2.2}

(i) All polyominoes $c$ with a fixed number of monominoes minimizing $\mathcal{T}(c)$ are singlecomponent monotone polyominoes of minimal perimeter, which include the standard polyominoes.

(ii) If the number of monominoes is $\ell^{2}, \ell^{2}-1, \ell(\ell-1)$ or $\ell(\ell-1)-1$ for some $\ell \in \mathbb{N} \backslash\{1\}$, then the standard polyominoes are the only minimizers of $\mathcal{T}(c)$.

Proof In the proof we assume without loss of generality that the polyomino consists of a single cluster $c$.

(i) The proof uses projection. Pick any non-monotone cluster $c$. Let

$$
\tilde{c}=\left(\pi_{2} \circ \pi_{1}\right)(c),
$$

where $\pi_{2}$ and $\pi_{1}$ denote the vertical, respectively, the horizontal projection of $c$. The effect of vertical and horizontal projection is illustrated in Fig. 8. By construction, $\tilde{c}$ is a monotone polyomino (see e.g. the statement on Ferrers diagrams in the proof of Alonso and Cerf [1], Theorem 2.2).

Suppose first that $Q(c)=0$. Then $\mathcal{T}(c)=2 P(c)+4$. Since $c$ is not monotone, we have $P(\tilde{c})<P(c)$, and so $c$ is not a minimizer of $\mathcal{T}(c)$.

Suppose next that $Q(c) \geq 1$. Since

$$
P(c)=\sum_{i=1}^{\ell_{2}(c)} v_{i}(c)+\sum_{j=1}^{\ell_{1}(c)} h_{j}(c)
$$

and every hole belongs to at least one row and one column, we have

$$
P(c) \geq 2\left[\ell_{1}(c)+\ell_{2}(c)\right]+4 Q(c) .
$$

On the other hand, since $\tilde{c}$ is a monotone polyomino, we have $v_{i}(\tilde{c})=h_{j}(\tilde{c})=2$ for all $i$ and $j$, and so

$$
P(\tilde{c})=2\left[\ell_{1}(\tilde{c})+\ell_{2}(\tilde{c})\right] .
$$

Moreover, since $\ell_{1}(\tilde{c}) \leq \ell_{1}(c)$ and $\ell_{2}(\tilde{c}) \leq \ell_{2}(c)$, we can combine (2.7-2.8) to get

$$
P(\tilde{c})-P(c) \leq-4 Q(c) .
$$

Using (2.9), we obtain

$$
\begin{aligned}
\mathcal{T}(\tilde{c})-\mathcal{T}(c) & =[2 P(\tilde{c})+4]-[2 P(c)+4-4 Q(c)] \\
& =2[P(\tilde{c})-P(c)]+4 Q(c) \leq-4 Q(c) \leq-4<0,
\end{aligned}
$$


Fig. 9 The circled boundary particle of type 1 belongs to: (a) class 1; (b) class 2; (c) class 3; (d) class 4

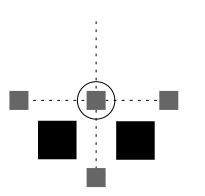

(a)

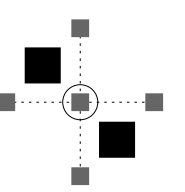

(b)

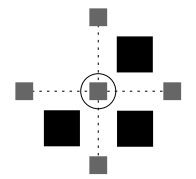

(c)

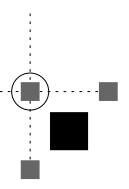

(d)

and so $c$ is not a minimizer of $\mathcal{T}(c)$.

(ii) We saw in the proof of (i) that if $c$ is a minimizer of $\mathcal{T}(c)$, then $c$ is monotone, and hence does not contain holes and minimizes $P(c)$. The claim therefore follows from Alonso and Cerf [1], Corollary 3.7, which states that if the number of monominoes is $\ell^{2}, \ell^{2}-1, \ell(\ell-1)$ or $\ell(\ell-1)-1$ for some $\ell \in \mathbb{N} \backslash\{1\}$, then the standard polyominoes are the only minimizers of $P(c)$.

\subsection{Relation Between $\mathcal{T}$ and the Number of Missing Bonds in 2-Tiled Clusters}

In this section we consider 2-tiled clusters and link the number of particles of type 1 and type 2 to the number of active bonds and the geometric quantity $\mathcal{T}$ considered in Sect. 2.3. Recall from Sect. 2.2, item 2, that $B(c)$ is the number of active bonds in $c$.

Lemma 2.3 For any 2-tiled cluster $c$ (i.e., $c \in \mathcal{V}_{\star, n_{2}}^{4 n_{2}}$ for some $\left.n_{2}\right), 4 n_{1}(c)=B(c)+\mathcal{T}(c)$ and $4 n_{2}(c)=B(c)$.

Proof The claim of the lemma is equivalent to the affirmation that $\mathcal{T}(c)=M(c)$ with $M(c)$ the number of missing bonds in $c$. Indeed, informally, for every unit perimeter two bonds are lost with respect to the four bonds that would be incident to each particle of type 1 if it were saturated, while one bond is lost at each convex corner and one bond is gained at each concave corner. Hence (2.3) yields the claim.

Formally, let $p$ be a particle of type $1, B(p)$ the number of bonds incident to $p$, and $M(p)=4-B(p)$ the number of missing bonds of $p$. Consider the set of particles of type 1 at the boundary of a 2-tiled cluster, i.e., the set of non-saturated particles of type 1. Each of these particles belongs to one of four classes (see Fig. 9, and recall the definition of 12-bar in Sect. 2.2, item 5):

class 1: $p$ has two neighboring particles of type 2 belonging to the same 12-bar.

class 2: $p$ has two neighboring particles of type 2 belonging to different 12-bars.

class 3: $p$ has three neighboring particles of type 2 .

class 4: $p$ has one neighboring particle of type 2 .

Let $M_{k}(c)$ be the number of missing bonds of particles of class $k$ in cluster $c$, and $A_{k}(c)$ the number of edges incident to particles of class $k$ in cluster $c$. Then

$$
\begin{array}{llll}
M_{1}(c)=2, & A_{1}(c)=2 ; & M_{2}(c)=2, & A_{2}(c)=4 ; \\
M_{3}(c)=1, & A_{3}(c)=2 ; & M_{4}(c)=3, & A_{4}(c)=2 .
\end{array}
$$

Let $N_{k}(c)$ be the number of particles of class $k$ of type 1 in cluster $c$. Observing that a cluster has two concave corners per particle of class 2, one concave corner per particle of class 3 and one convex corner per particle of class 4 , we can write

$$
\mathcal{T}(c)=2 P(c)-2 N_{2}(c)-N_{3}(c)+N_{4}(c) .
$$


Fig. 10 Representation of $\Lambda$ and $\Lambda^{-}$in dual coordinates, and the two possible ground states for $L=15$ when the cluster is:

(a) even; (b) odd. Note that sites in $\partial^{-} \Lambda$ are empty because there is no interaction in $\partial^{-} \Lambda$. Also note that the top-left and bottom-right corners of $\Lambda$ and $\Lambda^{-}$in dual coordinates do not correspond to a site of $\mathbb{Z}^{2}$

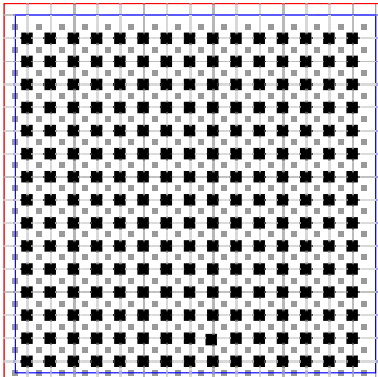

(a)

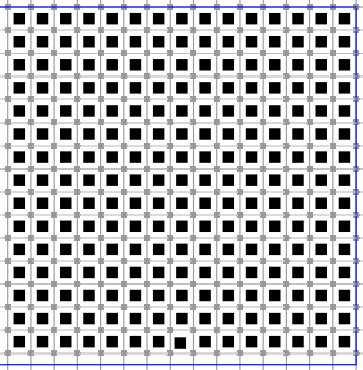

(b)

Since the dual perimeter of a cluster is equal to its total number of dual edges, we have

$$
2 P(c)=\sum_{k=1}^{4} A_{k}(c) N_{k}(c)=2 N_{1}(c)+4 N_{2}(c)+2 N_{3}(c)+2 N_{4}(c)
$$

(the sum counts each edge of the 2-tile twice). The total number of missing bonds, on the other hand, is

$$
M(c)=\sum_{k=1}^{4} M_{k}(c) N_{k}(c)=2 N_{1}(c)+2 N_{2}(c)+N_{3}(c)+3 N_{4}(c) .
$$

Combining (2.12-2.14), we arrive at $\mathcal{T}(c)=M(c)$.

\section{Proof of Theorem 1.3: Identification of $\mathcal{X}_{\text {meta }}$}

Recall that $\Lambda^{-}$(the part of $\Lambda$ where particles interact) is an $\left(L+\frac{1}{2}\right) \times\left(L+\frac{1}{2}\right)$ dual square with $L>2 \ell^{\star}$. Let $\eta_{\text {stab }}, \eta_{\text {stab }}^{\prime}$ be the configurations consisting of a 2 -tiled dual square of size $L$ with even parity, respectively, odd parity. (Note: Of the four corners of the $\left(L+\frac{1}{2}\right) \times$ $\left(L+\frac{1}{2}\right)$ dual square two diagonally opposite corners are empty since they do not correspond to a site of $\mathbb{Z}^{2}$.) These two configurations have the same energy. Theorem 1.3 says that $\mathcal{X}_{\text {stab }}=\left\{\eta_{\text {stab }}, \eta_{\text {stab }}^{\prime}\right\}=\boxplus$. Section 3.1 contains two lemmas about 2-tiled configurations with minimal energy. Section 3.2 uses these two lemmas to prove Theorem 1.3. (See also Fig. 10.)

\subsection{Standard Configurations Are Minimizers Among 2-Tiled Configurations}

Lemma 3.1 Within $\mathcal{V}_{\star, n_{2}}^{4 n_{2}}$, the standard configurations achieve the minimal energy.

Proof Recall from item 2 in Sect. 2.2 that

$$
H(\eta)=\Delta_{1} n_{1}(\eta)+\Delta_{2} n_{2}(\eta)-U B(\eta)
$$

In $\mathcal{V}_{\star, n_{2}}^{4 n_{2}}$ both $n_{2}$ and $B=4 n_{2}$ are fixed, and hence $\min _{\eta \in \mathcal{V}_{\star, n_{2}}^{4 n_{2}}} H(\eta)$ is attained at a configuration minimizing $n_{1}$. By Lemma 2.3, if $\eta \in \mathcal{V}_{\star, n_{2}}^{4 n_{2}}$, then

$$
n_{1}(\eta)=\frac{1}{4}[B(\eta)+\mathcal{T}(\eta)], \quad n_{2}(\eta)=\frac{1}{4} B(\eta) .
$$


Fig. 11 A standard

configuration with $\ell=7, \zeta=1$

and $k=5$

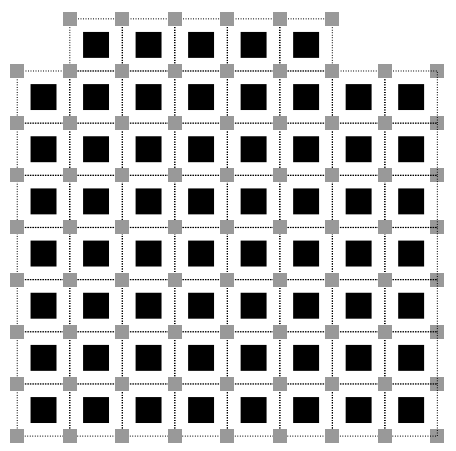

Hence, to minimize $n_{1}(\eta)$ we must minimize $\mathcal{T}(\eta)$. The claim therefore follows from Lemma 2.2(i).

For a standard configuration the computation of the energy is straightforward. For $\ell \in \mathbb{N}$, $\zeta \in\{0,1\}$ and $k \in \mathbb{N}_{0}$ with $k \leq \ell+\zeta$, let $\eta^{\ell, \zeta, k}$ denote the standard configuration consisting of an $\ell \times(\ell+\zeta)$ (quasi-)square with a bar of length $k$ attached to one of its longest sides (see Fig. 11).

Lemma 3.2 The energy of $\eta^{\ell, \zeta, k}$ is (recall (1.15))

$$
H\left(\eta^{\ell, \zeta, k}\right)=-\varepsilon[\ell(\ell+\zeta)+k]+\Delta_{1}\left[\ell+(\ell+\zeta)+1+1_{\{k>0\}}\right] .
$$

Proof Note that $P\left(\eta^{\ell, \zeta, k}\right)=2\left[\ell+(\ell+\zeta)+1_{\{k>0\}}\right]$ and $Q\left(\eta^{\ell, \zeta, k}\right)=0$, so that

$$
\mathcal{T}\left(\eta^{\ell, \zeta, k}\right)=4\left[\ell+(\ell+\zeta)+1+1_{\{k>0\}}\right]
$$

Also note that

$$
B\left(\eta^{\ell, \zeta, k}\right)=4[\ell+(\ell+\zeta)+k],
$$

because all particles of type 2 are saturated. However, by (3.1-3.2), we have

$$
H\left(\eta^{\ell, \zeta, k}\right)=-\frac{1}{4} \varepsilon B\left(\eta^{\ell, \zeta, k}\right)+\frac{1}{4} \mathcal{T}\left(\eta^{\ell, \zeta, k}\right) \Delta_{1},
$$

and so the claim follows by combining (3.4-3.6).

Note that the energy increases by $\Delta_{1}-\varepsilon$ (which is $>0$ if and only if $\ell^{\star} \geq 2$ by (1.14)) when a bar of length $k=1$ is added, and decreases by $\varepsilon$ each time the bar is extended. Note further that

$$
H\left(\eta^{\ell, 1,0}\right)-H\left(\eta^{\ell, 0,0}\right)=\Delta_{1}-\ell \varepsilon, \quad H\left(\eta^{\ell+1,0,0}\right)-H\left(\eta^{\ell, 1,0}\right)=\Delta_{1}-(\ell+1) \varepsilon,
$$

which show that the energy of a growing sequence of standard configurations goes up when $\ell<\ell^{\star}$ and goes down when $\ell \geq \ell^{\star}$. The highest energy is attained at $\eta^{\ell^{\star}-1,1,1}$, which is the critical droplet in Fig. 4.

It is worth noting that $H\left(\eta_{s}^{2 e^{\star}, 0,0}\right)<0$, i.e., the energy of a dual square of side length $2 \ell^{\star}$ is lower than the energy of $\square$. This is why we assumed $L>2 \ell^{\star}$, to allow for $H(\boxplus)<H(\square)$. 


\subsection{Stable Configurations}

In this section we use Lemmas 3.1-3.2 to prove Theorem 1.3.

Proof Let $\eta$ denote any configuration in $\mathcal{X}_{\text {stab }}$. Below we will show that:

(A) $\eta$ does not contain any particle in $\partial^{-} \Lambda$.

(B) $\eta$ is a 2-tiled configuration, i.e., $\eta \in \mathcal{V}_{\star, n_{2}}^{4 n_{2}}$ for some $n_{2}\left(=n_{2}(\eta)\right)$.

Once we have (A) and (B), we observe that $\eta$ cannot contain a number of 2-tiles larger than $L^{2}$. Indeed, consider the tile support of $\eta$. Since $\Lambda^{-}$is an $\left(L+\frac{1}{2}\right) \times\left(L+\frac{1}{2}\right)$ dual square, if the tile support of $\eta$ fits inside $\Lambda^{-}$, then so does the dual circumscribing rectangle of $\eta$. But any rectangle of area $\geq L^{2}$ has at least one side of length $L+1$. Hence $n_{2}(\eta) \leq L^{2}$, and therefore the number of 2-tiles in $\eta$ is at most $L^{2}$. By Lemmas 3.1-3.2, the global minimum of the energy is attained at the largest dual quasi-square that fits inside $\Lambda^{-}$, since $L>2 \ell^{\star}$. We therefore conclude that $\eta \in\left\{\eta_{\text {stab }}, \eta_{\text {stab }}^{\prime}\right\}$, which proves the claim.

Proof of (A) Since in $\partial^{-} \Lambda$ particles do not feel any interaction but have a positive energy cost, removal of a particle from $\partial^{-} \Lambda$ always lowers the energy.

Proof of $(B)$ We note the following three facts:

(1) $\eta$ does not contain isolated particles of type 1 .

(2) $\partial^{-} \Lambda^{-}$does not contain any particle of type 2 .

(3) All particles of type 2 in $\eta$ have all their neighboring sites occupied by a particle.

For (1), simply note that the configuration obtained from $\eta$ by removing isolated particles has lower energy. For (2), note that particles in $\partial^{-} \Lambda^{-}$have at most two active bonds. Therefore, if $\eta$ would have a particle of type 2 in $\partial^{-} \Lambda^{-}$, then the removal of that particle would lower the energy, because $\Delta_{2}-\Delta_{1}>2 U$ and $\Delta_{1}>0$ (recall (1.13)) imply $\Delta_{2}>2 U$. For (3), note that if a particle of type 2 has an empty neighboring site, then the addition of a particle of type 1 at this site lowers the energy, because $\Delta_{1}<U$ (recall (1.13)).

We can now complete the proof of (B) as follows. The constraint $\Delta_{2}-\Delta_{1}>2 U$ implies that any particle of type 2 in $\eta$ must have at least three neighboring sites occupied by a particle of type 1 . Indeed, the removal of a particle of type 2 with at most two active bonds lowers the energy. But the fourth neighboring site must also be occupied by a particle of type 1 . Indeed, suppose that this site would be occupied by a particle of type 2 . Then this particle would have at most three active bonds. Consider the configuration $\tilde{\eta}$ obtained from $\eta$ after replacing this particle by a particle of type 1 . Then $B(\tilde{\eta})-B(\eta) \geq-2, n_{1}(\tilde{\eta})-n_{1}(\eta)=$ 1 and $n_{2}(\tilde{\eta})-n_{2}(\eta)=-1$. Consequently, $H(\tilde{\eta})-H(\eta) \leq \Delta_{1}-\Delta_{2}+2 U<0$. Hence, any particle of type 2 in $\eta$ must be saturated.

\section{Proof of Theorem 1.5: Identification of $\Gamma^{\star}=\Phi(\square, \boxplus)$}

In Sect. 4.1 we prove Theorem 1.5 subject to the following lemma.

Lemma 4.1 For any $n_{2} \leq L^{2}$, the configurations of minimal energy with $n_{2}$ particles of type 2 belong to $\mathcal{V}_{\star, n_{2}}^{4 n_{2}}$, i.e., are 2-tiled configurations.

The proof of this lemma is given in Sect. 4.2. 


\subsection{Proof of Theorem 1.5 Subject to Lemma 4.1}

Proof For $\mathcal{Y} \subset \mathcal{X}$, define the external boundary of $\mathcal{Y}$ by $\partial \mathcal{Y}=\left\{\eta \in \mathcal{X} \backslash \mathcal{Y}: \exists \eta^{\prime} \in \mathcal{Y}, \eta \leftrightarrow \eta^{\prime}\right\}$ and the bottom of $\mathcal{Y}$ by $\mathcal{F}(\mathcal{Y})=\arg \min _{\eta \in \mathcal{Y}} H(\eta)$. According to Manzo, Nardi, Olivieri and Scoppola [5], Sect. 4.2, $\Phi(\square, \boxplus)=\min _{\eta \in \partial \mathcal{B}} H(\eta)$ for $\mathcal{B} \subset \mathcal{X}$ any (!) set with the following properties:

(I) $\mathcal{B}$ is connected via allowed moves, $\square \in \mathcal{B}$ and $\boxplus \notin \mathcal{B}$.

(II) There is a path $\omega^{\star}: \square \rightarrow \boxplus$ such that $\left\{\arg \max _{\eta \in \omega^{\star}} H(\eta)\right\} \cap \mathcal{F}(\partial \mathcal{B}) \neq \emptyset$.

Thus, our task is to find such a $\mathcal{B}$ and compute the lowest energy of $\partial \mathcal{B}$.

For (I), choose $\mathcal{B}$ to be the set of all configurations $\eta$ such that $n_{2}(\eta) \leq \ell^{\star}\left(\ell^{\star}-1\right)+1$. Clearly this set is connected, contains $\square$ and does not contain $\boxplus$.

For (II), choose $\omega^{\star}$ as follows. A particle of type 2 is brought inside $\Lambda\left(\Delta H=\Delta_{2}\right)$, moved to the origin and is saturated by four times bringing a particle of type $1(\Delta H=$ $\left.\Delta_{1}\right)$ and attaching it to the particle of type $2(\Delta H=-U)$. After this first 2-tile has been completed, $\omega^{\star}$ follows a sequence of increasing 2-tiled dual quasi-squares. The passage from one quasi-square to the next is obtained by adding a 12-bar to one of the longest sides, as follows. First a particle of type 2 is brought inside $\Lambda\left(\Delta H=\Delta_{2}\right)$ and is attached to one of the longest sides of the quasi-square $(\Delta H=-2 U)$. Next, twice a particle of type 1 is brought inside the box $\left(\Delta H=\Delta_{1}\right)$ and is attached to the (not yet saturated) particle of type 2 $(\Delta H=-U)$ in order to complete a 2-tiled protuberance. Finally, the 12-bar is completed by bringing a particle of type 2 inside $\Lambda\left(\Delta H=\Delta_{2}\right)$, moving it to a concave corner $(\Delta H=$ $-3 U)$, and saturating it with a particle of type $1\left(\Delta H=\Delta_{1}\right.$, respectively, $\left.\Delta H=-U\right)$. It is obvious that $\omega^{\star}$ eventually hits $\boxplus$. The path $\omega^{\star}$ is referred to as the reference path for the nucleation.

Call $\eta^{\star}$ the configuration in $\omega^{\star}$ consisting of an $\ell^{\star} \times\left(\ell^{\star}-1\right)$ quasi-square, a 2-tiled protuberance attached to one of its longest sides, and a free particle of type 2 (see Fig. 12; there are many choices for $\omega^{\star}$ depending on where the 2-tiled protuberances are added; all these choices are equivalent). Note that, in the notation of Lemma 3.2, $\eta^{\star}=\eta^{\ell^{\star}-1,1,1}+\mathrm{fp}[2]$, where $+\mathrm{fp}[2]$ denotes the addition of a free particle of type 2 in $\partial^{-} \Lambda$. Observe that:

(a) $\omega^{\star}$ exits $\mathcal{B}$ via the configuration $\eta^{\star}$;

(b) $\eta^{\star} \in \mathcal{F}(\partial \mathcal{B})$;

(c) $\eta^{\star} \in\left\{\arg \max _{\eta \in \omega^{\star}} H(\eta)\right\}$.

Observation (a) is obvious, while (b) follows from Lemmas 3.1 and 4.1. To see (c), note the following: (1) The total energy difference obtained by adding a 12-bar of length $\ell$ on the side of a 2-tiled cluster is $\Delta H$ (adding a 12-bar) $=\Delta_{1}-\varepsilon \ell$, which changes sign at $\ell=\ell^{\star}$ (recall (3.7)); (2) The configurations of maximal energy in a sequence of growing quasisquares are those where a free particle of type 2 enters the box after the 2-tiled protuberance has been completed. Thus, within energy barrier $2 \Delta_{1}+2 \Delta_{2}-4 U=4 U-\varepsilon$ the 12-bar is completed downwards in energy. This means that, after configuration $\eta^{\star}$ is hit, the dynamics can reach the 2-tiled dual square of $\ell^{\star} \times \ell^{\star}$ while staying below the energy level $H\left(\eta^{\star}\right)$. Since all 2-tiled dual quasi-squares larger than $\ell^{\star} \times\left(\ell^{\star}-1\right)$ have an energy smaller than that of the 2-tiled dual quasi-square $\ell^{\star} \times\left(\ell^{\star}-1\right)$ itself, the path $\omega^{\star}$ does not again reach the energy level $H\left(\eta^{\star}\right)$.

Because of (a-c), we have $\Phi(\square, \boxplus)=H\left(\eta^{\star}\right)$. To complete the proof, use Lemma 3.2 to compute

$$
H\left(\eta^{\star}\right)=H\left(\eta^{\ell^{\star}-1,1,1}+\mathrm{fp}[2]\right)=-\varepsilon\left[\ell^{\star}\left(\ell^{\star}-1\right)+1\right]+\Delta_{1}\left(2 \ell^{\star}+1\right)+\Delta_{2} .
$$


Fig. 12 A critical

configuration $\eta^{\star}$. This is the dual version of the critical droplet in Fig. 4.

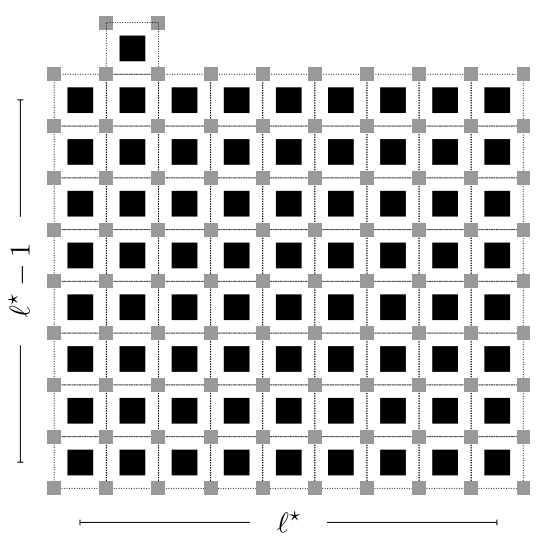

\subsection{Proof of Lemma 4.1}

The proof of Lemma 4.1 is carried out in two steps. In Sect. 4.2.1 we show that the claim holds for single-cluster configurations with a fixed number of particles of type 2 . In Sect. 4.2.2 we extend the claim to general configurations with a fixed number of particles of type 2 .

\subsubsection{Single Clusters of Minimal Energy Are 2-Tiled Clusters}

Lemma 4.2 For any single-cluster configuration $\eta \in \mathcal{V}_{\star, n_{2}} \backslash \mathcal{V}_{\star, n_{2}}^{4 n_{2}}$ there exists a configuration $\tilde{\eta} \in \mathcal{V}_{\star, n_{2}}^{4 n_{2}}$ such that $H(\tilde{\eta})<H(\eta)$.

Proof Pick any $\eta \in \mathcal{V}_{\star, n_{2}} \backslash \mathcal{V}_{\star, n_{2}}^{4 n_{2}}$. Every neighboring site of a particle of type 2 in the cluster is either empty or occupied by a particle of type 1 , and there is at least one non-saturated particle of type 2 . Since $\eta$ consists of a single cluster, $\tilde{\eta}$ can be constructed in the following way:

- $\tilde{\eta}(i)=\eta(i)$ for all $i \in \operatorname{supp}(\eta)$.

- $\tilde{\eta}(j)=1$ for all $j \notin \operatorname{supp}(\eta)$ such that there exists an $i \sim j$ with $\eta(i)=2$.

Since

$$
\begin{aligned}
& H(\eta)=\Delta_{1} n_{1}(\eta)+\Delta_{2} n_{2}(\eta)-U B(\eta), \\
& H(\tilde{\eta})=\Delta_{1} n_{1}(\tilde{\eta})+\Delta_{2} n_{2}(\tilde{\eta})-U B(\tilde{\eta}),
\end{aligned}
$$

and $n_{2}(\eta)=n_{2}(\tilde{\eta})$, we have

$$
H(\tilde{\eta})-H(\eta)=\Delta_{1}\left[n_{1}(\tilde{\eta})-n_{1}(\eta)\right]-U[B(\tilde{\eta})-B(\eta)] .
$$

By construction, $B(\tilde{\eta})-B(\eta) \geq n_{1}(\tilde{\eta})-n_{1}(\eta)>0$. Since $0<\Delta_{1}<U$ (recall (1.13)), it follows from (4.3) that $H(\tilde{\eta})<H(\eta)$. 


\subsubsection{Configurations of Minimal Energy with Fixed Number of Particles of Type 2}

Lemma 4.3 For any $n_{2}$ and any configuration $\eta \in \mathcal{V}_{\star, n_{2}}$ consisting of at least two clusters, any configuration $\eta^{\star}$ such that $\eta^{\star}$ is a single cluster, $\eta^{\star} \in \mathcal{V}_{\star, n_{2}}^{4 n_{2}}$ and $\eta^{\star}$ is a standard configuration satisfies $H\left(\eta^{\star}\right)<H(\eta)$.

Proof Let $\eta \in \mathcal{V}_{\star, n_{2}}$ be a configuration consisting of $k>1$ clusters, labeled $c_{1}, \ldots, c_{k}$. Let $\eta^{n_{2}\left(c_{i}\right)}$ denote any standard configuration with $n_{2}\left(c_{i}\right)$ particles of type 2. By Lemmas 3.1 and 4.2, we have

$$
H(\eta)=\sum_{i=1}^{k} H\left(c_{i}\right) \geq \sum_{i=1}^{k} H\left(\eta^{n_{2}\left(c_{i}\right)}\right) .
$$

By Lemma 2.3, we have (recall (1.15))

$$
\begin{aligned}
\sum_{i=1}^{k} H\left(\eta^{n_{2}\left(c_{i}\right)}\right) & =\sum_{i=1}^{k}\left[\Delta_{1} n_{1}\left(\eta^{n_{2}\left(c_{i}\right)}\right)+\Delta_{2} n_{2}\left(\eta^{n_{2}\left(c_{i}\right)}\right)-U B\left(\eta^{n_{2}\left(c_{i}\right)}\right)\right] \\
& =\sum_{i=1}^{k}\left[\Delta_{1}\left\{n_{2}\left(\eta^{n_{2}\left(c_{i}\right)}\right)+\frac{1}{4} \mathcal{T}\left(\eta^{n_{2}\left(c_{i}\right)}\right)\right\}+\Delta_{2} n_{2}\left(\eta^{n_{2}\left(c_{i}\right)}\right)-U 4 n_{2}\left(\eta^{n_{2}\left(c_{i}\right)}\right)\right] \\
& =\sum_{i=1}^{k}\left[-\varepsilon n_{2}\left(\eta^{n_{2}\left(c_{i}\right)}\right)+\frac{1}{4} \Delta_{1} \mathcal{T}\left(\eta^{n_{2}\left(c_{i}\right)}\right)\right]
\end{aligned}
$$

But from Lemma 2.2 it follows that

$$
\sum_{i=1}^{k} \mathcal{T}\left(\eta^{n_{2}\left(c_{i}\right)}\right)>\mathcal{T}\left(\eta^{\sum_{i=1}^{k} n_{2}\left(c_{i}\right)}\right),
$$

where $\eta^{\sum_{i=1}^{k} n_{2}\left(c_{i}\right)}$ denotes any standard configuration with $\sum_{i=1}^{k} n_{2}\left(c_{i}\right)=n_{2}(\eta)$ particles of type 2. Combining (4.4-4.6), we arrive at

$$
H(\eta)>-\varepsilon n_{2}(\eta)+\frac{1}{4} \Delta_{1} \mathcal{T}\left(\eta^{n_{2}(\eta)}\right)=H\left(\eta^{n_{2}(\eta)}\right) .
$$

\section{Proof of Theorem 1.4: Upper Bound on $V_{\eta}$ for $\eta \notin\{\square$, $⿴ 囗\}$}

In this section we show that for any configuration $\eta \notin\{\square, \boxplus\}$ it is possible to find a path $\omega: \eta \rightarrow \eta^{\prime}$ with $\eta^{\prime} \in\{\square, \boxplus\}$ such that $\max _{\xi \in \omega} H(\xi) \leq H(\eta)+V^{\star}$ with $V^{\star} \leq 10 U-\Delta_{1}$ and $\eta^{\prime} \in I_{\eta}$. By Definition 1.1(c-e), this implies that $V_{\eta} \leq V^{\star}$ for all $\eta \notin\{\square$, $\boxplus\}$ and therefore settles Theorem 1.4.

Section 5.3 describes an energy reduction algorithm to find $\omega$. Roughly, the idea is that if $\eta$ contains only "subcritical clusters", then these clusters can be removed one by one to reach $\square$, while if $\eta$ contains some "supercritical cluster", then this cluster can be taken as a stepping stone to construct a path to $\boxplus$ that goes via a sequence of increasing rectangles. In 


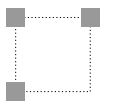

(i)

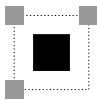

(ii)

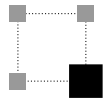

(iii)

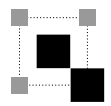

(iv)

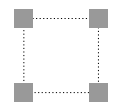

(v)

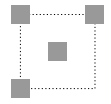

(vi)

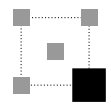

(vii)

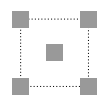

(viii)

Fig. 13 Possible tiles with at least three junction sites occupied by a particle of type 1

particular, the supercritical cluster is first extended to a 2-tiled rectangle touching the northboundary of $\Lambda$, after that it is extended to a 2-tiled rectangle touching the west-boundary and the east-boundary of $\Lambda$, and finally it is extended to $\boxplus$.

To carry out this task, six energy reduction mechanisms are needed, which are introduced and explained in Sect. 5.2:

- Moving unit holes inside 2-tiled clusters (Sect. 5.2.1).

- Adding and removing 12-bars from lattice-connecting rectangles (Sect. 5.2.2).

- Changing bridges into 12-bars (Sect. 5.2.3).

- Maximally expanding 2-tiled rectangles (Sect. 5.2.4).

- Merging adjacent 2-tiled rectangles (Sect. 5.2.5).

- Removing subcritical clusters (Sect. 5.2.6).

Each of Sects. 5.2.1-5.2.6 states a definition and a lemma, and uses these to prove a proposition about the relevant energy reduction mechanism. The six propositions thus obtained will be crucial for the energy reduction algorithm in Sect. 5.3.

In Sect. 5.1 we begin by defining beams and pillars, which are needed throughout Sect. 5.2.

\subsection{Beams and Pillars}

Lemma 5.1 Let $\eta$ be a configuration containing a tile that has at least three junction sites occupied by a particle of type 1 . Then the configuration $\eta^{\prime}$ obtained from $\eta$ by turning $t$ into a 2-tile satisfies $H\left(\eta^{\prime}\right) \leq H(\eta)$.

Proof Without loss of generality we may assume that $\eta\left(t_{a}\right)=\eta\left(t_{b}\right)=\eta\left(t_{d}\right)=1$, and that $\eta^{\prime}$ is the configuration in Fig. $6(\mathrm{~d})$, i.e., $\eta^{\prime}\left(t_{a}\right)=\eta^{\prime}\left(t_{b}\right)=\eta^{\prime}\left(t_{c}\right)=\eta^{\prime}\left(t_{d}\right)=1, \eta^{\prime}\left(t_{e}\right)=2$. The following eight cases are possible (see Fig. 13 and recall (1.13)):

(i) $\left(\eta\left(t_{c}\right), \eta\left(t_{e}\right)\right)=(0,0)$. One particle of type 1 and one particle of type 2 are added, and at least four new bonds are activated: $\Delta H \leq \Delta_{1}+\Delta_{2}-4 U<0$.

(ii) $\left(\eta\left(t_{c}\right), \eta\left(t_{e}\right)\right)=(0,2)$. One particle of type 1 is added, and one new bond is activated: $\Delta H=\Delta_{1}-U<0$.

(iii) $\left(\eta\left(t_{c}\right), \eta\left(t_{e}\right)\right)=(2,0)$. One particle of type 2 is moved to another site without deactivating any bonds, after which case (ii) applies.

(iv) $\left(\eta\left(t_{c}\right), \eta\left(t_{e}\right)\right)=(2,2)$. One particle of type 2 with at most three active bonds is replaced by one particle of type 1 with at least one active bond: $\Delta H \leq \Delta_{1}-\Delta_{2}+$ $2 U<0$.

(v) $\left(\eta\left(t_{c}\right), \eta\left(t_{e}\right)\right)=(1,0)$. One particle of type 2 is added, and four new bonds are activated: $\Delta H=\Delta_{2}-4 U<0$.

(vi) $\left(\eta\left(t_{c}\right), \eta\left(t_{e}\right)\right)=(0,1)$. One particle of type 1 is moved to another site without deactivating any active bond, one particle of type 2 is added, and at least four new bonds are activated: $\Delta H \leq \Delta_{2}-4 U<0$.

(vii) $\left(\eta\left(t_{c}\right), \eta\left(t_{e}\right)\right)=(2,1)$. Two particles are exchanged without deactivating any bonds: $\Delta H \leq 0$. 


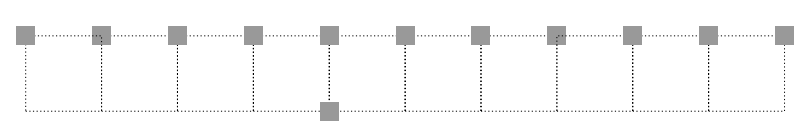

Fig. 14 A south-pillared horizontal beam of length 10 with a west-section of length 4 and an east-section of length 6

(viii) $\left(\eta\left(t_{c}\right), \eta\left(t_{e}\right)\right)=(1,1)$. One particle of type 1 is replaced by a particle of type 2 , and four new bonds are activated: $\Delta H=\Delta_{2}-\Delta_{1}-4 U<0$.

Definition 5.2 A beam of length $\ell$ is a row (or column) of $\ell+1$ particles of type 1 at dual distance 1 of each other. A pillar is a particle of type 1 at dual distance 1 of the beam not located at one of the two ends of the beam. The particle in the beam sitting next to the pillar divides the beam into two sections. The lengths of these two sections are $\geq 0$ and sum up to $\ell$. The support of a pillared beam is the union of all the tile supports. The support consists of three rows (or columns) of sites — an upper, middle and lower row (or column)—which are referred to as roof, center and basement (see Fig. 14).

Note that a beam can have more than one pillar. Lemma 5.1 implies the following.

Corollary 5.3 Let $\eta$ be a configuration containing a pillared beam $\tilde{b}$ such that $\operatorname{supp}(\tilde{b})$ is not 2-tiled. Then the configuration $\eta^{\prime}$ obtained from $\eta$ by 2-tiling $\operatorname{supp}(\tilde{b}) \operatorname{satisfies} H\left(\eta^{\prime}\right) \leq$ $H(\eta)$.

\subsection{Six Energy Reduction Mechanisms}

\subsubsection{Moving Unit Holes Inside 2-Tiled Clusters}

In this section we show how a unit hole can move inside a 2-tiled cluster. In particular, we show that such motion is possible within an energy barrier $6 U$ by changing the configuration only locally.

Definition 5.4 A set of sites $S$ inside $\Lambda$ obtained from a $4 \times 4$ square after removing the four corner sites is called a slot.

Given a slot $S$, we assign a label to each of the 12 sites in $S$ as in Fig. 15(a): first clockwise in the center of $S$ and then clockwise on the boundary of $S$. We call the pairs $\left(S_{1}, S_{3}\right)$ and $\left(S_{2}, S_{4}\right)$ slot-conjugate sites.

Lemma 5.5 Let $S$ be a slot, and let $\eta_{0}$ be any configuration such that all particles in $S$ have the same parity. Without loss of generality this parity may be taken to be even, so that $\eta\left(S_{1}\right)=0$ and $\eta\left(S_{3}\right)=2$. Let $\eta_{1}$ be the configuration obtained from $\eta$ by interchanging the states of $S_{1}$ and $S_{3}$. Then $H\left(\eta_{0}\right)=H\left(\eta_{1}\right)$, and there exists a path $\omega: \eta_{0} \rightarrow \eta_{1}$ that never exceeds the energy level $H\left(\eta_{0}\right)+6 U$.

Proof Without loss of generality we take $\eta_{0}$ as in Fig. 15(b-c). Let $a \rightarrow b$ denote the motion of a particle from site $a$ to site $b$. For the path $\omega$ we choose the following sequence of moves: $S_{4} \rightarrow S_{1} ; S_{3} \rightarrow S_{4} ; S_{2} \rightarrow S_{3} ; S_{1} \rightarrow S_{2} ; S_{4} \rightarrow S_{1} ; S_{3} \rightarrow S_{4}$. The first three moves and the second three moves each are a rotation by $\frac{\pi}{2}$ of the subconfiguration at the sites 


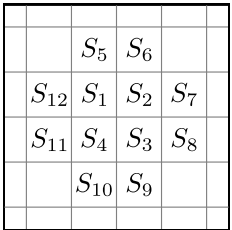

(a)

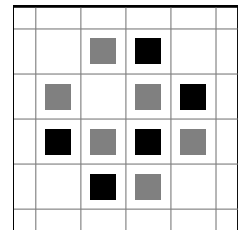

(b)

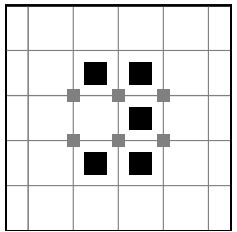

(c)

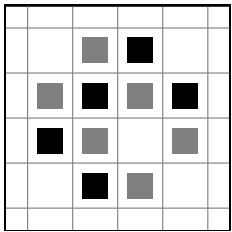

(d)

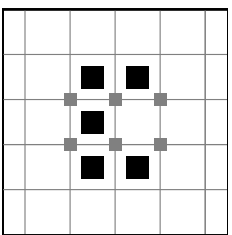

(e)

Fig. 15 (a) Labeling of the sites in the slot (standard representation); (b) example of $\eta_{0}$ in the slot (standard representation); (c) example of $\eta_{0}$ in the slot (dual representation). (d) $\eta_{1}$ in the slot (standard representation); (e) of $\eta_{1}$ in the slot (dual representation)

Fig. 16 Motion of a unit hole inside a 2-tiled cluster

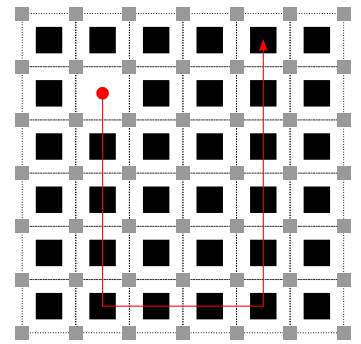

$S_{1}, S_{2}, S_{3}, S_{4}$. Note that all configurations in $\omega$ have the same number of particles of each type and hence the changes in energy only depend on the change in the number of active bonds. Let $M_{R F}$ be the loss of the number of active bonds between the rotating particles and the fixed particles, and $M_{R}$ the loss of the number of active bonds between the rotating particles. We must show that $M_{R F}+M_{R} \leq 6$ during the six moves. To that end, we first observe that $M_{R F} \leq 6$, since the total number of active bonds between the rotating particles and the fixed particles is at most 6 (see Fig. 15(b)), and that $M_{R F}=6$ only after the first three moves are completed, i.e., when the configuration is such that all the rotating particles have a different parity with respect to the parity they had in configuration $\eta_{0}$ (recall that particles with different parity cannot share a bond). Next we observe that, by the choice of $\omega$, the value of $M_{R}$ can only be 0 or 1 , and that $M_{R}=0$ after the first three moves are completed.

Lemma 5.5 implies the following.

Proposition 5.6 Let $\eta$ be a 2-tiled configuration with a unit hole. Then the configuration $\eta^{\prime}$ obtained from $\eta$ by moving the unit hole elsewhere satisfies $H\left(\eta^{\prime}\right)=H(\eta)$ and $\Phi\left(\eta, \eta^{\prime}\right) \leq$ $H(\eta)+6 U$.

A possible $6 U$-path for a unit hole inside a 2-tiled cluster is given in Fig. 16. This path is obtained through an iteration of local moves as explained in Fig. 15.

\subsubsection{Adding and Removing 12-Bars from Lattice-Connecting Rectangles}

Lemma 5.7 Let $\eta$ be a configuration consisting of a single 2-tiled lattice-connecting rectangle. Then the configuration $\eta^{\prime}$ obtained from $\eta$ by, respectively, 


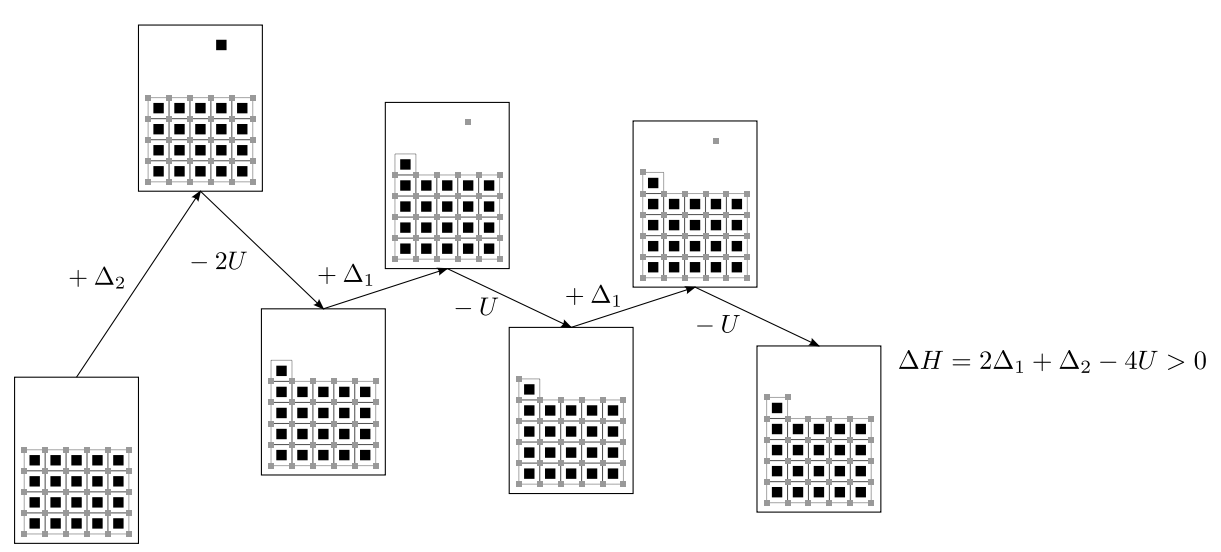

Fig. 17 A 2-tiled protuberance is added to a side of a dual rectangle within energy barrier $\Delta_{2}$

1. adding a 12-bar of length $\ell \geq \ell^{\star}$,

2. adding a 12-bar of length $\ell<\ell^{\star}$,

3. removing a 12 -bar of length $\ell \geq \ell^{\star}$,

4. removing a 12-bar of length $\ell<\ell^{\star}$,

satisfies, respectively,

1. $H\left(\eta^{\prime}\right)<H(\eta)$ and $\Phi\left(\eta, \eta^{\prime}\right) \leq H(\eta)+2 \Delta_{1}+2 \Delta_{2}-4 U$,

2. $H\left(\eta^{\prime}\right)>H(\eta)$ and $\Phi\left(\eta, \eta^{\prime}\right) \leq H(\eta)+2 \Delta_{1}+2 \Delta_{2}-4 U$,

3. $H\left(\eta^{\prime}\right)>H(\eta)$ and $\Phi\left(\eta, \eta^{\prime}\right) \leq H(\eta)+(\ell-2) \varepsilon+4 U-\Delta_{1}$,

4. $H\left(\eta^{\prime}\right)<H(\eta)$ and $\Phi\left(\eta, \eta^{\prime}\right) \leq H(\eta)+(\ell-2) \varepsilon+4 U-\Delta_{1}$.

Proof Recall the computations in Sects. 3.1 and 4.1.

Adding a 12-Bar Adding a 12-bar of length $\ell$ on a lattice-connecting side of a 2-tiled rectangle (i.e., a side such that all the particles of type 1 on that side are lattice-connecting) can be done in two steps: (i) initiate the 12-bar by adding a 2-tiled protuberance (see Fig. 17); (ii) complete the 12-bar by adding a 2-tile (in a "corner") $\ell-1$ times (see Fig. 18). This can be achieved within energy barrier $\Delta H=2 \Delta_{1}+2 \Delta_{2}-4 U$ by following the same moves as the reference path $\omega^{\star}$ described in Sect. 4.1. The energy difference due to the extra 12-bar of length $\ell$ is $\Delta H(\ell)=\Delta_{1}-\ell \varepsilon$, which changes sign at $\ell=\ell^{\star}$.

Removing a 12-Bar Removing a 12-bar of length $\ell$ from a lattice-connecting rectangle can be done by following the reverse of the path used to add a 12-bar: (i) remove $\ell-1$ times a 2-tile from a bar; (ii) remove the last 2-tiled protuberance. This can be achieved within energy barrier $\Delta H(\ell)=(\ell-2) \varepsilon+4 U-\Delta_{1}$. If the cluster consists of one 12-bar only, then the path just described leaves $\ell+1$ free particles of type 1 inside $\Lambda$, which can be removed (free of energy cost) afterwards.

We use Lemma 5.7 to build a northern rectangle on top of a 12-bar as follows.

Definition 5.8 Let $b$ denote the vertical coordinate of the sites lying on the north-side of $\partial^{-} \Lambda^{-}$. For a given 2-tiled rectangle $r$ in $\Lambda^{-}$, let $b_{r}$ denote the vertical coordinate of the 


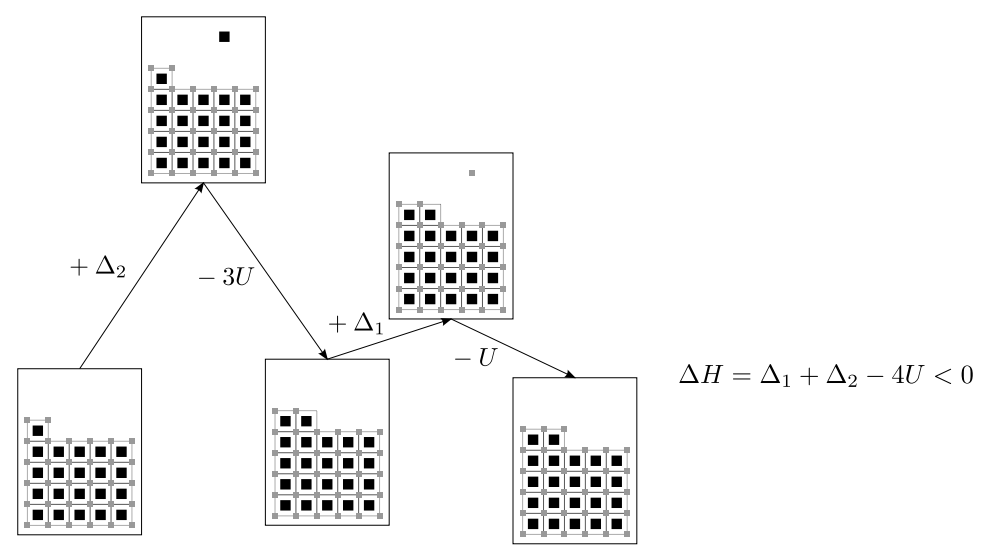

Fig. 18 A 2-tile is added in a corner between 2-tiles within a energy barrier $\Delta_{2}$

Fig. 19 A stable bridge of length 6

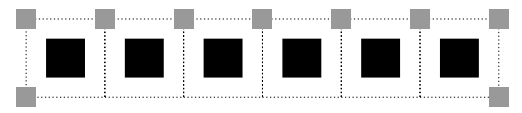

northern-most particles of type 1 in $r$. Then $r$ is said to be touching the north-side of $\partial^{-} \Lambda^{-}$ if $b_{r}=b$ or $b_{r}=b-\frac{1}{2}$.

In words, a 2-tiled rectangle is said to be touching the north-side of $\partial^{-} \Lambda^{-}$if it is not possible to add a 12-bar on the north-side within $\Lambda^{-}$. Rectangles touching the south-, east- or westside of $\Lambda^{-}$are defined similarly.

Let $\bar{b}$ be a horizontal 12-bar of length $\ell$, i.e., a 2-tiled $\ell \times 1$ rectangle. Suppose that all sites above $\bar{b}$ are vacant. Then it is possible to successively add horizontal 12-bars, say $m$ in total, on top of $\bar{b}$ until the north side of the rectangle grown in this way touches the north-side of $\Lambda^{-}$. The 2-tiled rectangle with $m+1$ rows and $\ell$ columns such that $\bar{b}$ is its lower-most horizontal 12-bar is denoted by $\sqcap(\bar{b})$ and is called the northern rectangle of $\bar{b}$.

Lemma 5.7 implies the following.

Proposition 5.9 Let $\eta$ be a configuration containing a horizontal 12-bar $\bar{b}$ of length $\ell \geq \ell^{\star}$. Then the configuration $\eta^{\prime}$ obtained from $\eta$ by building $\sqcap(\bar{b})$ satisfies $H\left(\eta^{\prime}\right)<H(\eta)$ and $\Phi\left(\eta, \eta^{\prime}\right) \leq H(\eta)+2 \Delta_{1}+2 \Delta_{2}-4 U$.

\subsubsection{Changing Bridges into 12-Bars}

Definition 5.10 A (south-)bridge $b$ consists of a beam $\tilde{b}$ and two (south-)pillars at the outer-most sites of the (south-)basement of $\tilde{b}$. The (south-)support of $b$ coincides with the (south-)support of $\tilde{b}$. If each of the central sites of the tiles of the (south-)support of the bridge is occupied by a particle of type 2, then the bridge is said to be stable (see Fig. 19).

Clearly, a 12-bar is a stable bridge. North-, east- and west-bridges are defined in a similar way.

Given a bridge $b$, let $\bar{b}$ denote the 12-bar obtained by 2-tiling $b$. Lemma 5.1 implies the following. 
Lemma 5.11 Let $\eta$ be a configuration containing a bridge $b$ whose support is not 2-tiled. Then the configuration $\eta^{\prime}$ obtained from $\eta$ by changing $b$ to $\bar{b}$ satisfies $H\left(\eta^{\prime}\right)<H(\eta)$.

Lemma 5.11 leads us to the following.

Proposition 5.12 Let $\eta$ be a configuration containing a (south-)bridge $b$ whose (south-) support is not 2-tiled such that the particles of its beam are lattice-connecting. Then the configuration $\eta^{\prime}$ obtained from $\eta$ by 2-tiling supp $(b)$ satisfies $H\left(\eta^{\prime}\right)<H(\eta)$ and $\Phi\left(\eta, \eta^{\prime}\right) \leq$ $H(\eta)+4 U+\Delta_{1}$.

Proof Let the (south-)bridge $b$ have length $\ell$. Label the $\ell+1$ sites of its (south-)basement as $s_{0}, s_{1}, \ldots, s_{\ell}$, from the left to the right. In order to show that $\operatorname{supp}(b)$ can be 2-tiled within energy barrier $4 U+\Delta_{1}$, it is enough to show that within the same energy barrier a particle of type 1 can be brought to a site of the basement of $b$ (from the left) that is empty or is occupied by a particle of type 2 . Without loss of generality $s_{1}$ may be assumed to be such a site. The configuration thus obtained has an energy that is at most the energy of the original configuration (see Lemma 5.1). The claim follows by noting that the particles of type 1 at the extremal sites $s_{1}$ and $s_{\ell}$ are the two pillars of a (south-)bridge of length $\ell-1$ whose basement consists of the sites $s_{1}, s_{2}, \ldots, s_{\ell}$.

It remains to show how a particle of type 1 can be brought to site $s_{1}$. Label the site northwest of $s_{1}$ by $v_{1}$, and the site north-east of $v_{1}$ by as $v_{2}$. Two cases need to be distinguished:

(1) If $\eta\left(s_{1}\right)=0$, then, by the same argument as in the proof of Lemma 5.5, it is easy to show that the particle of type 1 at $v_{2}$ can be moved to $s_{1}$ (to obtain a configuration $\bar{\eta}$ with $H(\bar{\eta}) \leq H(\eta)$ ) without exceeding energy level $H(\eta)+4 U$. The configuration $\eta^{\prime}$ is reached within an energy barrier $\Delta_{1}$ by bringing a particle of type 1 inside $\Lambda$ and moving it to $v_{2}$.

(2) If $\eta\left(s_{1}\right)=2$, then consider the following path. First detach $(\Delta H=2 U)$ and remove $\left(\Delta H=-\Delta_{1}\right)$ the particle of type 1 at $v_{2}$, and afterwards detach $(\Delta H=2 U)$ and remove ( $\Delta H=-\Delta_{2}$ ) the particle of type 2 at $v_{3}$. Next, move the particle of type 2 at site $s_{1}$ to site $v_{1}\left(\Delta H \leq 0\right.$; this particle has at most 2 active bonds when it sits at $\left.s_{1}\right)$, and finally bring a particle of type $1\left(\Delta H=\Delta_{1}\right)$ to site $v_{2}(\Delta H=-2 U)$. Call this configuration $\bar{\eta}$. Note that $H(\bar{\eta})<H(\eta)$, since effectively a particle of type 2 with at most two active bonds has been removed, and $\Phi\left(\eta, \eta^{\prime}\right)=H(\eta)+4 U+\Delta_{1}$. Finally, observe that $\eta^{\prime}$ is the same configuration as $\eta$ in Case (1).

\subsubsection{Maximally Expanding 2-Tiled Rectangles}

The mechanism presented in this section, which is called north maximal expansion of a 2-tiled rectangle, is such that it can be applied to a 2-tiled rectangle whose north-side is lattice-connecting (even though this condition is not restrictive). South, east and west maximal expansion of a 2-tiled cluster are analogous.

Definition 5.13 The north maximal expansion comes in two phases: a growing phase and a smoothing phase.

(i) The growing phase consists of the following three steps repeated cyclically:

1. If the particles of type 1 on the south-side of the rectangle, either at the beginning or obtained after step 3 , constitute a south-pillared beam $\tilde{b}_{s}$, then change $\operatorname{supp}\left(\tilde{b}_{s}\right)$ into a 12-bar. 


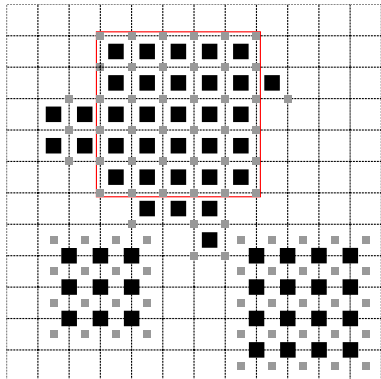

(a)

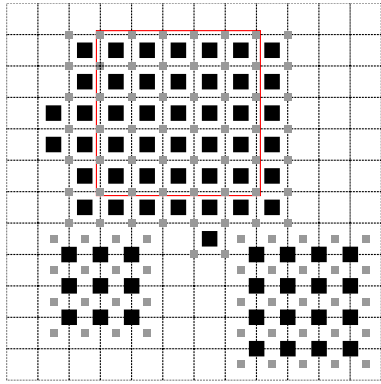

(d)

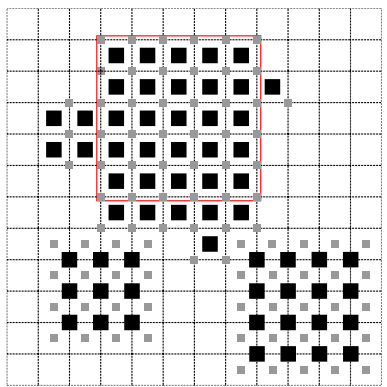

(b)

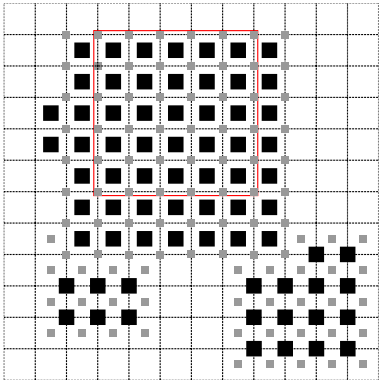

(e)

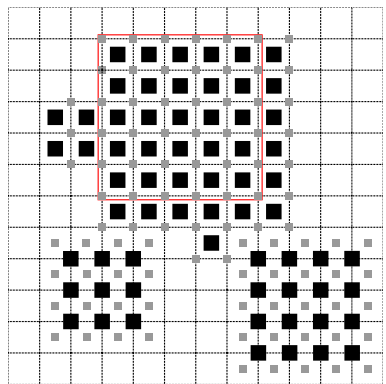

(c)

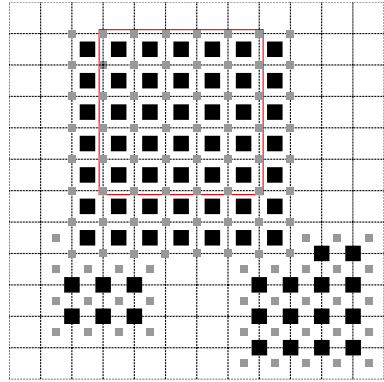

(f)

Fig. 20 Example of north maximal expansion of a 2-tiled rectangle. The outcome of the steps of the growing phase are represented in pictures (b-e), while the outcome of the smoothing phase is represented in picture (f)

2. If the particles of type 1 on the east-side of the rectangle, obtained after step 1 , constitute an east-pillared beam $\tilde{b}_{e}$, then change $\operatorname{supp}\left(\tilde{b}_{e}\right)$ into a 12-bar.

3 . If the particles of type 1 on the west-side of the rectangle, obtained after step 2 , constitute a west-pillared beam $\tilde{b}_{w}$, then change $\operatorname{supp}\left(\tilde{b}_{w}\right)$ into a 12-bar.

The growing phase ends after three consecutive steps leave the configuration unchanged.

(ii) The smoothing phase consists of removing all the particles of type 2 that are adjacent to the ones on the sides of the rectangle that is built during the growing phase. Note that these particles have at most two active bonds (otherwise it would be possible to identify another pillared beam), and therefore removal of these particles lowers the energy.

The outcome of the north maximal expansion (see Fig. 20) of a 2-tiled rectangle is again a 2-tiled rectangle, containing the old rectangle and such that the northern-most 12-bar of the new rectangle has the same vertical coordinate.

Given a 2-tiled rectangle $r$, let $\mathcal{R}^{\top}(r)$ denote the north maximal expansion of $r$. Corollary 5.3 implies the following.

Lemma 5.14 Let $\eta$ be a configuration containing a 2-tiled rectangle. Then the configuration $\eta^{\prime}$ obtained from $\eta$ via (north) maximal expansion of this 2-tiled rectangle satisfies then $H\left(\eta^{\prime}\right) \leq H(\eta)$.

Lemma 5.14 leads us to the following. 
Proposition 5.15 Let $\eta$ be a configuration containing a 2-tiled rectangle $r$ whose northside is lattice-connecting. Then the configuration $\eta^{\prime}$ obtained from $\eta$ after replacing $r$ by $\mathcal{R}^{\top}(r)$ satisfies $H\left(\eta^{\prime}\right) \leq H(\eta)$ and $\Phi\left(\eta, \eta^{\prime}\right) \leq H(\eta)+10 U-\Delta_{1}$.

Proof If $\mathcal{R}^{\top}(r)=r$, then there is nothing to prove. Therefore suppose that $r$ is such that one its sides is a pillared beam. Without loss of generality we may assume that the south-side of $r$ is a beam $\tilde{b}$ with a south-pillar. We must show that the south-support of $\tilde{b}$ can be turned into a 12-bar within energy barrier $10 U-\Delta_{1}$.

Since $\operatorname{supp}(\tilde{b})$ is not a 12-bar, a pillar can be chosen in such a way that at least one of the 2-tiles of the support the pillar belongs to (i.e., the first tile of each section of the support, counting from the pillar) is not a 2-tile. Without loss of generality we let this tile be the first tile of the right-section and call it $t$. Let $v$ denote the tile adjacent to the right site of $v$. In the following, the term superficial refers to tiles that are in the top tile-bar of the rectangle. In analogy with the proof of Lemma 5.1, several cases need to be considered (we stick to the order in Fig. 13).

(i) $\left(\eta\left(t_{c}\right), \eta\left(t_{e}\right)\right)=(0,0)$. A particle of type 2 has to be brought to site $t_{e}$ and a particle of type 1 to site $t_{c}$. First bring a particle of type 2 to site $t_{e}$, to reach a configuration $\hat{\eta}$, and then proceed as in Case (ii). As we will see in Case (ii), since $H(\hat{\eta})=H(\eta)-$ $3 U+\Delta_{2}$, the second part of the path can be completed without exceeding energy level $H(\eta)+6 U+\Delta_{2}$. To reach configuration $\hat{\eta}$, move the particle of type 2 of the 2-tile above $t$ to site $t_{e}$ to reach a configuration called $\eta^{\prime}$. This can be done without exceeding energy level $H(\eta)+6 U$. Note that $H\left(\eta^{\prime}\right)=H(\eta)+U$. The unit hole that has been created at the central site of the tile above $t$ has to be filled. This can be done (see Lemma 5.5) by first moving the unit hole until it becomes superficial (configuration $\tilde{\eta}$ with energy $H(\tilde{\eta})=H\left(\eta^{\prime}\right)$ ) without exceeding energy level $H\left(\eta^{\prime}\right)+6 U$, and then filling this unit hole with a particle of type 2 within energy level $H\left(\eta^{\prime}\right)+U-\Delta_{1}+$ $\Delta_{2}=H(\eta)+2 U-\Delta_{1}+\Delta_{2}$. Thus, $\eta^{\prime}$ can be reached without exceeding energy barrier $6 U+\Delta_{2}$.

(ii) $\left(\eta\left(t_{c}\right), \eta\left(t_{e}\right)\right)=(0,2)$. A particle of type 1 has to be brought to site $t_{c}$. Depending on the state of site $v_{e}$, there are three cases.

(a) Site $v_{e}$ is occupied by a particle of type 2. Move the particle of type 1 at site $t_{b}$ to site $t_{c}$, to reach a configuration $\eta^{\prime}$ with energy $H\left(\eta^{\prime}\right) \leq H(\eta)+2 U$ within an energy barrier of $6 U$. The vacancy at site $t_{b}$ can be moved (again by Lemma 5.5) to the north-side of the rectangle within energy barrier $6 U$, to reach a configuration $\hat{\eta}$ with $H(\hat{\eta}) \leq H(\eta)$, and then filled with an extra particle of type 1 . Thus, $\eta^{\prime}$ can be reached without exceeding energy level $H(\eta)+8 U$.

(b) Site $v_{e}$ is empty. Move the particle of type 1 at site $t_{b}$ to site $v_{e}(\Delta H \leq 3 U)$, and then to site $t_{d}(\Delta H=0)$. Call this configuration $\eta^{\prime}$, and note that $H\left(\eta^{\prime}\right) \leq$ $H(\eta)+2 U$. Arguing as above, we see that the vacancy at site $t_{b}$ can be filled without exceeding the energy level $H(\eta)+9 U$.

(c) Site $v_{e}$ is occupied by a particle of type 1 . Observe that the particle of type 1 at $t_{b}$ has $k \leq 3$ active bonds and the particle of type 2 at $v_{e}$ has $m \leq 2$ active bonds. It is possible to move the particle at site $v_{e}$ to site $t_{c}(\Delta H=(m-k) U)$, and then the particle at site $t_{b}$ to site $v_{c}(\Delta H=(k-m) U)$. The configuration $\eta^{\prime}$, reached within energy barrier $(k-m) U$, has energy $H\left(\eta^{\prime}\right) \leq H(\eta)+k U$. Again, the vacancy at site $t_{b}$ has to be filled with a particle of type 1 . This can be done without exceeding the energy level $H(\eta)+(6+k) U \leq H(\eta)+9 U$.

(iii) $\left(\eta\left(t_{c}\right), \eta\left(t_{e}\right)\right)=(2,0)$. The particle of type 2 at site $t_{c}$ is moved to site $t_{e}$ without increasing the energy. Then argue as in Case (ii). 
(iv) $\left(\eta\left(t_{c}\right), \eta\left(t_{e}\right)\right)=(2,2)$. The particle of type 2 at site $t_{c}$ has to be replaced by a particle of type 1 . Remove the particle of type 2 at $t_{e}$. To do this, first create a superficial unit hole (which can be done within energy barrier $4 U-\Delta_{1}$ by creating a hole in a corner tile of the rectangle) and move this vacancy to site $t_{e}$. By Lemma 5.5, this can be achieved without exceeding energy level $H\left(\eta_{0}\right)+10 U-\Delta_{2}$. Then move the particle of type 2 at site $t_{c}$ to site $t_{e}(\Delta H \leq 0)$. Call $\eta^{\prime}$ the configuration that is reached in this way. Note that $H\left(\eta^{\prime}\right) \leq H(\eta)-\Delta_{2}+3 U$. To bring a particle of type 1 to site $t_{c}$, argue as in Case (ii), to arrive at $H(\hat{\eta}) \leq H(\eta)+12 U-\Delta_{2}$.

(v) $\left(\eta\left(t_{c}\right), \eta\left(t_{e}\right)\right)=(1,0)$. A particle of type 2 has to be brought to site $t_{e}$. Move the unit hole at $t_{e}$ to the top tile-bar of the rectangle. This does not change the energy of the configuration and can be done within energy barrier $6 U$ by Proposition 5.6. The task reduces to filling a superficial unit hole on the surface of the cluster with a particle of type 2 . This can be achieved within energy barrier $U+\Delta_{2}-\Delta_{1}$. Therefore the maximal energy level reached in this case is $H(\eta)+6 U$.

(vi) $\left(\eta\left(t_{c}\right), \eta\left(t_{e}\right)\right)=(0,1)$. Move the particle of type 2 from site $t_{e}$ to site $t_{c}$. This move does not increase the energy of the configuration. Then proceed as in Case (v).

(vii) $\left(\eta\left(t_{c}\right), \eta\left(t_{e}\right)\right)=(2,1)$. The occupation numbers of sites $t_{c}$ and $t_{e}$ have to be exchanged. To do this, first remove the particle of type 1 at site $t_{b}$ to obtain a configuration $\eta^{\prime}$ with energy $H\left(\eta^{\prime}\right) \leq H(\eta)+3 U$ without exceeding the energy level $H(\eta)+10 U-\Delta_{1}$ (again use Lemma 5.5). Move the particle of type 1 from $t_{e}$ to $t_{b}(\Delta H<0)$ and the particle of type 2 from $t_{c}$ to $t_{e}(\Delta H=0)$. Call $\hat{\eta}$ the configuration that is reached in this way. Note that $H(\hat{\eta}) \leq H(\eta)+U-\Delta_{1}$. Proceed as in Case (ii) to conclude within energy barrier of $10 U-\Delta_{1}$.

(viii) $\left(\eta\left(t_{c}\right), \eta\left(t_{e}\right)\right)=(1,1)$. The particle of type 1 at site $t_{e}$ has to be replaced by a particle of type 2 . This can be done as follows. First the particle of type 1 sitting a site $t_{b}$ is removed. To achieve this, first remove a particle of type 1 at the north-side of the rectangle and then (use Lemma 5.5) move the vacancy to site $t_{b}$. The configuration that is reached, which we call $\eta^{\prime}$, is such that $H\left(\eta^{\prime}\right) \leq H(\eta)+3 U-\Delta_{1}$. Next, move the particle of type 1 at $t_{e}$ to site $t_{b}(\Delta H=0)$, to reach a configuration $\hat{\eta}$ whose energy is $H(\hat{\eta})=H(\eta)-\Delta_{1}$. Finally, argue as in Case (v), to arrive at $H(\hat{\eta}) \leq H(\eta)+$ $3 U-\Delta_{1}$.

Finally, note that (1.13) implies $\max \left\{6 U+\Delta_{2}, 10 U-\Delta_{1}, 12 U-\Delta_{2}\right\}=10 U-\Delta_{1}$.

By Lemma 5.1, $H\left(\eta^{\prime}\right) \leq H(\eta)$, and therefore the same argument can be used to show that all the right-sections of the support can be 2-tiled within the same energy barrier. The left-section can be 2-tiled analogously.

To conclude, it remains to be shown how particles of type 2, possibly adjacent to one side of the rectangle, can be removed from $\Lambda$. Call $t$ the tile associated with the particle $p$ of type 2 that has to be removed ( $p$ sits at site $t_{e}$ ) and $v$ the tile adjacent to $t$ belonging to the rectangle. First bring a vacancy to site $v_{e}$ within energy barrier $10 U-\Delta_{2}$ (one way to achieve this has been described in Case (iv) above) and then move $p$ to site $v_{e}$ (see Lemma 5.5).

\subsubsection{Merging Adjacent 2-Tiled Rectangles}

Definition 5.16 A 12-bar $b_{1}$ of length $\ell$ of a cluster $c_{1}$ is said to be adjacent to a 12-bar $b_{2}$ of length $m \leq \ell$ of a cluster $c_{2}$ if there exist $m$ mutually disjoint pairs $\left(q_{1}^{i}, q_{2}^{i}\right)$ of particles of type 1 with $q_{1}^{i} \in b_{1}$ and $q_{2}^{i} \in b_{2}$ such that $u\left(q_{1}^{i}\right)-u\left(q_{2}^{i}\right)=v$ with $\|v\|=\frac{1}{2} \sqrt{2}$ for $i=$ $1, \ldots, m$. The vector $v$ is called the offset of $b_{2}$ with respect to $b_{1}$. The tiles in $b_{1}$ have 


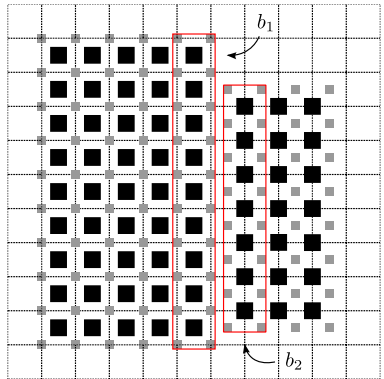

(a) $\eta_{0}$

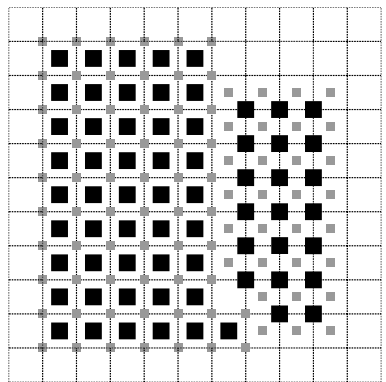

(d)

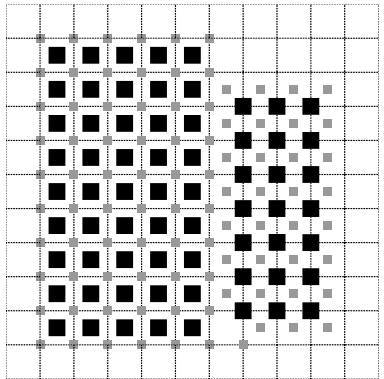

(b)

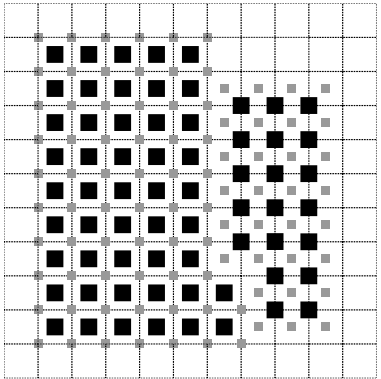

(e)

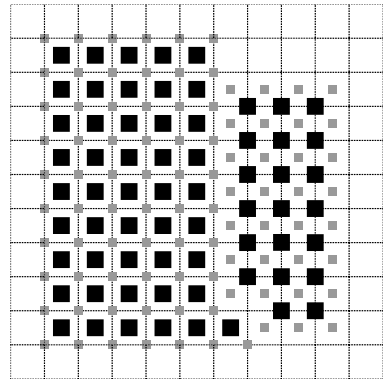

(c)

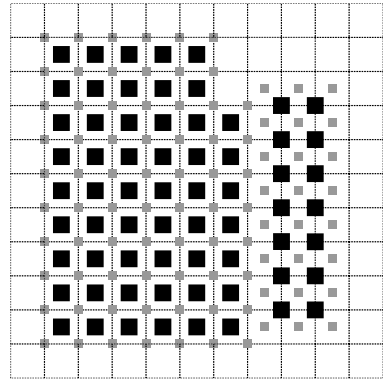

(f) $\eta_{1}$

Fig. 21 The sliding of $b_{2}$ onto $b_{1}$

a different parity than the tiles in $b_{2}$. The particles $q_{1}^{i} \in b_{1}, i=1, \ldots, m$, are called the external particles of $b_{1}$ with respect to $b_{2}$, and the particles $q_{2}^{i} \in b_{2}, i=1, \ldots, m$, are called the external particles of $b_{2}$ with respect to $b_{1}$.

Proposition 5.17 Let $\eta$ be a configuration that contains two adjacent 2-tiled rectangles. Then the configuration $\eta^{\prime}$ obtained by "merging" these two rectangles satisfies $H\left(\eta^{\prime}\right)=$ $H(\eta)$ and $\Phi\left(\eta, \eta^{\prime}\right) \leq H(\eta)+2 U-\Delta_{1}$.

Proof Given two adjacent bars $b_{1}$ and $b_{2}$ with offset $v=\left(v_{1}, v_{2}\right)$ in a configuration $\eta$, we want to define the sliding of $b_{2}$ onto $b_{1}$ along $v$. The resulting configuration $\eta^{\prime}$ is such that all the particles of type 2 originally in $b_{2}$ are slid by $\left(v_{1}, v_{2}\right)$ with respect to their position in $\eta$, and all the external particles of type 1 of $b_{2}$ with respect to $b_{1}$ are slid by $\left(v_{1},-v_{2}\right)$ when the two bars are horizontal and by $\left(-v_{1}, v_{2}\right)$ when the two bars are vertical. Via the sliding, the $m$ 2-tiles in $b_{2}$ are turned into $m$ 2-tiles with the same parity as the tiles in $b_{1}$. It is easy to see that $H\left(\eta^{\prime}\right)=H(\eta)$, since neither the total number of active bonds of the configuration nor the number of particles of each type is changed.

To describe the sliding of a bar onto another bar along a vector $v$, we may assume without loss of generality that the two bars are vertical and that the vector $v$ is equal to $\left(-\frac{1}{2},-\frac{1}{2}\right)$ (Fig. 21(a)). Start by moving the lower-most external particle of type 1 in $b_{2}$ over the vector $v^{\prime}=\left(\frac{1}{2},-\frac{1}{2}\right)$ (Fig. 21(b)). This leads to an increase by $U$ in energy. Then move the lowermost particle of type 2 over the vector $v$ (Fig. 21(c)). Since the number of deactivated bonds is equal to the number of new bonds activated, this move does not change the energy. Proceed by moving over the vector $v^{\prime}$ the second particle of type 1 from the bottom of the bar (Fig. 21(d)). This also is a move that does not change the energy. Afterwards, the second particle of type 2 from the top is moved over the vector $v$ (Fig. 21(e)). This sequence of 
Fig. 22 A pending dimer is the pair of particles circled in the picture

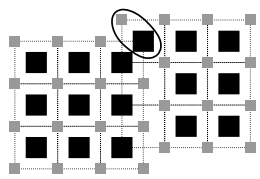

moves proceeds iteratively (without a change in energy) until the $m$-th particle of type 2 has been moved over the vector $v$. Finally, the $(m+1)$-st external particle of type 1 is moved over the vector $v^{\prime}$ (Fig. 21(f)). This move decreases the energy by $U$. Thus, $U$ is the energy barrier that must be overcome in order to realize the sliding of a 12-bar onto another 12-bar over the vector $v$.

It is clear that, given a configuration $\eta$ containing two 2-tiled rectangles $c_{1}$ (with vertical side length $\ell$ ) and $c_{2}$ (with vertical side length $m \leq \ell$ ) with offset $v$, it is possible to reduce $\eta$ to a configuration $\eta^{\prime}$ such that $c_{1}$ and $c_{2}$ are merged into of a single cluster by sliding one bar after another, without exceeding energy barrier $\Delta H=U$, provided the other clusters of $\eta$ do not interfere with this procedure. Sliding the last bar of $c_{2}$ we get an excess of free particles of type 1 , which can be removed from $\Lambda$, lowering the energy. In particular, the configuration $\eta^{\prime}$ obtained via the sliding of $c_{2}$ onto $c_{1}$ along $v$ without exceeding energy level $H(\eta)+U$ has energy $H\left(\eta^{\prime}\right)=H(\eta)-(m+1) \Delta_{1}$, since the two configurations consist of the same number of 2-tiles, and $\eta^{\prime}$ contains $m+1$ particles of type 1 less than $\eta$. Moreover, $\Phi\left(\eta, \eta^{\prime}\right)=H(\eta)+U$.

In the argument above, the first move consisted of moving down-right a particle of type 1 of $b_{2}$ to an empty site (say, site $i$ ). If in configuration $\eta$ site $i$ is occupied by a particle of type 1 , then the sliding of the vertical 12-bar can be realized by modifying the procedure as follows. First remove from the box the top-left particle of type 1 of $b_{2}$ sitting at site $j$ to reach a configuration with energy $H(\eta)+U-\Delta_{1}$ (which can be done without exceeding energy level $H(\eta)+U$ ). Then move to $j$ the particle of type 1 sitting at site $k=j+v=$ $j+\left(-\frac{1}{2},-\frac{1}{2}\right)$ in $\eta$, which increases the energy up to level $H(\eta)+2 U-\Delta_{1}$. Then site $k$ is filled with the particle of type 1 originally at site $k+\left(\frac{1}{2},-\frac{1}{2}\right)$ without an increase in energy. It is possible to continue in this way until the configuration obtained after the first step of the above case is reached. This configuration has energy $H(\eta)+U-\Delta_{1}$. Then proceed as in the above case until $b_{2}$ is slid onto $b_{1}$. This leads to a configuration with energy $H(\eta)-\Delta_{1}<H(\eta)$. In order to perform the (modified) sliding procedure, it is sufficient to assume that the north-side of rectangle $c_{2}$ is lattice-connecting.

\subsubsection{Removing Subcritical Clusters}

The cleaning mechanism defined in this section produces a configuration for which we have a certain control on the geometry of the constituent clusters. In particular, these clusters will be suitable for the application of the previous five energy reduction mechanisms. We begin by looking at pending dimers (see Fig. 22).

Definition 5.18 A pending dimer consists of two adjacent particles of different type such that the particle of type 1 is lattice-connecting and has only one active bond and the particle of type 2 has at most three active bonds.

Proposition 5.19 Let $\eta$ be a configuration containing pending dimers. Then there exists a configuration $\eta^{\prime}$ not containing pending dimers that satisfies $H\left(\eta^{\prime}\right)<H(\eta)$ and $\Phi\left(\eta, \eta^{\prime}\right) \leq$ $H(\eta)+3 U+\Delta_{2}$. 
Proof If the particle of type 2 has at most two active bonds, then simply remove the pending dimer. This reduces the energy, since two bonds are deactivated and a particle of each type is removed from $\Lambda\left(\Delta H \leq 2 U-\Delta_{1}-\Delta_{2}<0\right)$, and can be achieved within an energy barrier $2 U-\Delta_{1}$ along the following path: first detach $(\Delta H=U)$ and remove $\left(\Delta H=-\Delta_{1}\right)$ the particle of type 1 , then detach $(\Delta H \leq U)$ and remove $\left(\Delta H=-\Delta_{2}\right)$ the particle of type 2 .

If the particle of type 2 has three active bonds we have two cases:

(i) The fourth neighbor of the particle of type 2 of the pending dimer is empty. In this case $\eta^{\prime}$ is obtained by filling this empty site with a particle of type 1 in order to obtain a 2-tile, which lowers the energy since $\Delta_{1}<U$. To do this, temporarily remove the pending dimer as described above. This leads to a configuration $\tilde{\eta}$ with energy $H(\tilde{\eta})=$ $H(\eta)+3 U-\Delta_{1}-\Delta_{2}$ reached within energy barrier $3 U-\Delta_{1}$. Then bring a particle of type 1 to the designated site $\left(\Delta H \leq \Delta_{1}\right)$ and finally put back the dimer. The whole path is realized within energy barrier $3 U+\Delta_{2}$.

(ii) The fourth neighbor of the particle of type 2 is occupied by a particle of type 2 . In this case $\eta^{\prime}$ is the configuration such that the dimer is removed and the site originally occupied by the particle of type 2 of the dimer is occupied by a particle of type 1 . To obtain $\eta^{\prime}$ from $\eta$, remove the pending dimer (again, as above, within energy barrier $3 U-\Delta_{1}$ ), to reach a configuration $\tilde{\eta}$ with energy $H(\tilde{\eta})=H(\eta)+3 U-\Delta_{1}-\Delta_{2}$, and bring a particle of type 1 within energy barrier $\Delta_{1}$. To conclude, observe that $H\left(\eta^{\prime}\right)=$ $H(\eta)+2 U-\Delta_{2}<H(\eta)$.

The cleaning mechanism works as follows:

1. Remove all the lattice-connecting free particles from the configuration. After that repeat cyclically the following two steps:

2. Iteratively remove/transform all the lattice-connecting pending dimers.

3. Bring a particle of type 1 to any of the free sites adjacent to the lattice-connecting particles of type 2 .

Repeat the cleaning mechanism until the configuration is not affected anymore. Each of the three steps can be performed within energy barrier $3 U+\Delta_{2}$. Moreover, each step reduces the energy.

Lemma 5.20 The outcome of the cleaning mechanism is either a configuration such that the first particle encountered while scanning $\Lambda$ in the lexicographic order is a particle of type 1 belonging to a horizontal stable (south-)bridge, or the configuration $\square$.

Proof Call $q$ the first particle of $\Lambda$ in the lexicographic order. Recall that the dual coordinates of $q$ are denoted by $u(q)=\left(u_{1}(q), u_{2}(q)\right)$. Step 3 of the cleaning mechanism guarantees that $q$ is a particle of type 1 . The fact that $q$ is the first particle in the lexicographic order implies that: (i) all the sites above $u(q)$ are empty; (ii) all the sites with the same vertical coordinate as $q$ lying on the left of $q$ are empty as well. As a consequence of (ii), all the sites on the left of $q$ with vertical coordinate $u_{2}(q)-\frac{1}{2}$ are lattice-connecting and therefore cannot be occupied by a particle of type 2 . Since $q$ cannot be a free particle, the site with coordinates $\left(u_{1}(q)+\frac{1}{2}, u_{2}(q)-\frac{1}{2}\right)$ must be occupied by a particle $p$ of type 2 . Let $s(p)$ be the longest sequence of tiles adjacent to $t(p)$ such that the central site is occupied by a particle of type 2. Obviously, $p$ is the left-most particle of type 2 in $s(p)$. Call $\tilde{p}$ the last particle of type 2 in $s(p)$ and $\tilde{q}$ the particle of type 1 with coordinates $\left(u_{1}(p)+\frac{1}{2}, u_{2}(p)+\frac{1}{2}\right)$. (Note that $p$ and $\tilde{p}$ may coincide.) All the sites on the north-side of $s(p)$ are lattice-connecting and hence are occupied by a particle of type 1 . To conclude, observe that both $p$ and $\tilde{p}$ 
must be saturated, otherwise at least one of the pairs $(q, p)$ and $(\tilde{q}, \tilde{p})$ constitutes a pending dimer.

\subsection{Energy Reduction of a General Configuration: Proof of Theorem 1.4}

Fix any $\eta \notin\{\square, \boxplus\}$. In this section we will give a general procedure, called energy reduction algorithm, that allows us to construct a path $\omega: \eta \rightarrow \eta_{r}$ with $\eta_{r} \in\{\square$, $\boxplus\}$ such that $\max _{\xi \in \omega} H(\xi) \leq H(\eta)+V^{\star}$ with $V^{\star} \leq 10 U-\Delta_{1}$ and $H\left(\eta_{r}\right)<H(\eta)$. Note that if $\eta_{r}=\boxplus$, then $H\left(\eta_{r}\right)<H(\eta)$ because $\mathcal{X}_{\text {stab }}=\boxplus$. The construction uses the six energy reduction mechanisms described in Sects. 5.2.1-5.2.6 and relies on Propositions 5.6, 5.9, 5.12, $5.15,5.17,5.19$, which are the key results of these sections. The maximal energy barrier in these propositions is $10 U-\Delta_{1}$. Note: The energy reduction mechanisms in Sects. 5.2.2 and 5.2.3 concern single droplets far away from $\partial^{-} \Lambda$ and have an energy barrier not exceeding $4 U+\Delta_{1}<\Gamma^{\star}$ (see below (1.15)). For such configurations, the energy can be essentially reduced by saturating particles of type 2 and by adding and removing 12-bars. This explains the remark made in Sect. 1.4, item 4.

In the remainder of this section we call supercritical a 12-bar of length $\geq \ell^{\star}$. Similarly, we call supercritical a dual rectangle with both side lengths $\geq \ell^{\star}$.

Proof As a preliminary step, perform the cleaning mechanism. If the outcome is $\square$, then the claim is proven. Otherwise, let $b_{1}$ be the first bridge encountered in the lexicographic order (which exists by Lemma 5.20). This bridge can be turned into an 12-bar $\bar{b}_{1}$ (see Sect. 5.2.3). If the length of $b_{1}$ is $<\ell^{\star}$, then the 12-bar $\bar{b}_{1}$ can be removed, which lowers the energy (see Sect. 5.2.2). In this case, go back to performing the cleaning mechanism. Without loss of generality we may therefore assume that the length of $b_{1}$ is $>\ell^{\star}$.

By construction, all sites above $\bar{b}_{1}$ are empty, and therefore it is possible first to construct the 2-tiled rectangle $r_{1}=\sqcap\left(\bar{b}_{1}\right)$ within energy barrier $2 \Delta_{1}+2 \Delta_{2}-4 U$ (again lowering the energy), and then expand $r_{1}$ to the rectangle $R_{1}=\mathcal{R}^{\top}\left(r_{1}\right)$ (see Sect. 5.2.4). If the vertical side length of $R_{1}$ is $<\ell^{\star}$, then $R_{1}$ can be removed (lowering the energy), and it is possible to perform again the cleaning mechanism.

Therefore suppose that $R_{1}$ has both its side lengths $\geq \ell^{\star}$. In the remainder of the section we will show how to reach within energy barrier $10 U-\Delta_{1}$ a configuration containing a rectangle $R_{N W}$ touching both the north-side and the west-side of $\Lambda^{-}$whose support contains the support of $R_{1}$. Once this has been achieved, it is possible to argue for $R_{N W}$ in the same way as for $R_{1}$ in order to reach a configuration containing a rectangle $R_{N W E}$ touching the north-side, the east-side and the west-side of $\Lambda^{-}$whose support contains the support of $R_{N W}$. Repeating the same argument for $R_{N W E}$, it is possible to reach $\boxplus$.

The construction of $R_{N W}$ is obtained by using an algorithm called invasion of $R_{1}$, which is constructed with the help of techniques similar to the ones that were used to build $R_{1}$.

(A) Invasion of $R_{1} \quad$ See Fig. 23. Let $\left(a_{1}, b_{1}\right)$ be, respectively, the horizontal and the vertical coordinate of the left lower-most particle of $R_{1}$ (which is of type 1). Define $\Lambda\left(R_{1}\right) \subset \Lambda$ to be the set consisting of the sites whose vertical coordinate is $\geq b_{1}$ and horizontal coordinate is $<a_{1}$. In words, $\Lambda\left(R_{1}\right)$ contains the sites of $\Lambda$ on the left of $R_{1}$. Perform the cleaning mechanism (see Sect. 5.2.6) and scan $\Lambda\left(R_{1}\right)$ in the lexicographic order. Three cases are possible.

1. $\Lambda\left(R_{1}\right)$ is empty. Add, if possible ( $R_{1}$ might already be touching the west-boundary of $\Lambda^{-}$), 12-bars onto the left side of $R_{1}$ until the resulting cluster touches the west-boundary of $\Lambda^{-}$. 
2. The first horizontal bridge $b_{2}$ encountered in $\Lambda\left(R_{1}\right)$ has length $<\ell^{\star}$. Remove the particles of the (south)-support of the bridge, lowering the energy of the configuration, and restart the covering of $\Lambda\left(R_{1}\right)$.

3. The first horizontal bridge $b_{2}$ encountered in $\Lambda\left(R_{1}\right)$ has length $\geq \ell^{\star}$. As for $b_{1}$, first turn $b_{2}$ into the 12-bar $\bar{b}_{2}$, then build the 2-tiled rectangle $r_{2}=\sqcap\left(\bar{b}_{2}\right)$, after that expand $r_{2}$ to $R_{2}=\mathcal{R}^{\top}\left(r_{2}\right)$, and finally perform the cleaning mechanism. Note that the support of $R_{2}$ may cover (part or possibly all of) the support of $R_{1}$. This means that during the maximal expansion, some of the sites of $\operatorname{supp}\left(R_{1}\right)$ were in the support of the pillared beam that is going to be 2-tiled. Each time this happens, $R_{2}$ absorbs an entire vertical supercritical 12-bar of $R_{1}$ (see Sect. 5.2.4). Call $\tilde{R}_{1}$ what is left of $R_{1}$ after the maximal expansion of $R_{2}$. The following three cases are possible: (i) $\tilde{R}_{1}$ does not contain any particle $\left(\tilde{R}_{1}=\emptyset\right)$; (ii) $\tilde{R}_{1} \prec R_{1}$ (in the proper sense); (iii) $\tilde{R}_{1}=R_{1}$. In Case (ii), the rectangles $R_{2}$ and $\tilde{R}_{1}$ are necessarily adjacent (more precisely, the right-most 12-bar of $R_{2}$ is adjacent to the left-most 12-bar of $R_{1}$ ), whereas in Case (iii) the two rectangles may or may not be adjacent. Note that this implies that if $\tilde{R}_{1} \prec R_{1}$, then $R_{2}$ is necessarily supercritical. Obviously, if $\tilde{R}_{1} \neq \emptyset$, then it is again a 2 -tiled rectangle, and there are several possibilities.

(a) $R_{2}$ is not supercritical. This implies that $\tilde{R}_{1}=R_{1}$. Remove $R_{2}$ from $\Lambda$, put $R_{1}=\tilde{R}_{1}$ and restart the invasion of $R_{1}$.

(b) $R_{2}$ is supercritical and $\tilde{R}_{1}=\emptyset$. Change the name of $R_{2}$ to $R_{1}$ and restart the covering of $\Lambda\left(R_{1}\right)$.

(c) $R_{2}$ is supercritical and is adjacent to $\tilde{R}_{1}$. Note that both rectangles touch the northside of $\Lambda^{-}$. Call $R^{\max }$ the rectangle with the largest vertical length (in case of a tie, without loss of generality choose $R_{1}$ ) and call $R^{\mathrm{min}}$ the other rectangle. Slide $R^{\mathrm{min}}$ onto $R^{\max }$. This is possible because the smoothing phase of the maximal expansion (see Sect. 5.2.4) removes all the particles of type 2 that may interfere with the sliding of the 12-bars. Then perform again the maximal expansion of $R^{\max }$, i.e., the rectangle that has not been moved during the sliding. These steps bring the configuration to a rectangle whose support contains $\operatorname{supp}\left(R_{2}\right) \cup \operatorname{supp}\left(R_{1}\right) \cup \Lambda\left(R_{1}\right)$. Call this rectangle $R_{1}$ and restart the invasion of $R_{1}$.

(d) $R_{2}$ is supercritical and is not adjacent to $\tilde{R}_{1}$. This implies $\tilde{R}_{1}=R_{1}$. Start the invasion of $R_{2}$ (see below).

In order to complete the proof, it remains to show how the invasion of $R_{2}$ carries over. To that end, we introduce the following recursive algorithm realizing the invasion of $R_{i}$ for $i=2,3, \ldots$, etc.

(B) Invasion of $R_{i} \quad$ Call $\bar{R}_{i-1}$ what is left of $R_{i-1}$ after the invasion of $R_{i+1}$. There are three cases:

I. $\bar{R}_{i-1}=\emptyset$ (i.e., the support of $R_{i-1}$ is completely covered by $R_{i}$ ). Put $R_{i-1}=R_{i}$ and restart the invasion of $R_{i-1}$.

II. $\bar{R}_{i-1} \neq \emptyset$ and $R_{i}$ and $\bar{R}_{i-1}$ are adjacent. Call $R^{\max }$ the rectangle with the largest vertical side between $R_{i}$ and $\bar{R}_{i-1}$ (in case of a tie, without loss of generality choose $R^{\max }=$ $R_{i}$ ) and call $R^{\text {min }}$ the other rectangle. Slide $R^{\min }$ onto $R^{\max }$ and perform the maximal expansion of $R^{\max }$. Call $R_{i-1}$ the outcome of the maximal expansion of $R^{\mathrm{max}}$ and restart the invasion of $R_{i-1}$.

III. $\bar{R}_{i-1} \neq \emptyset$ and $R_{i}$ and $\bar{R}_{i-1}$ are not adjacent. If $R_{i}$ is on the left of $R_{i-1}$, then let $\left(a_{i}, b_{i}\right)$ denote, respectively, the horizontal and the vertical coordinate of the lower right-most particle (which is of type 1 ) of $R_{i}$, and call $\Lambda\left(R_{i}\right)$ the subset of $\Lambda\left(R_{i-1}\right)$ consisting 


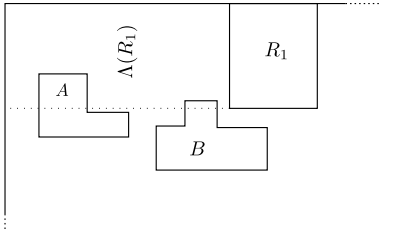

(a)

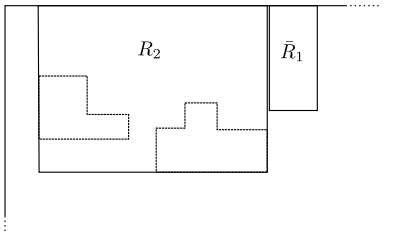

(d)

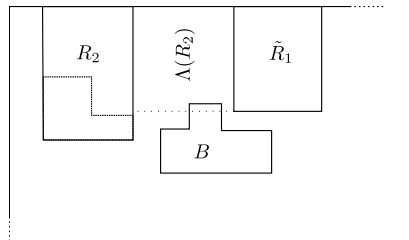

(b)

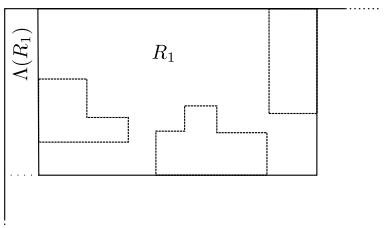

(e)

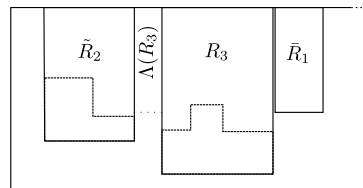

(c)

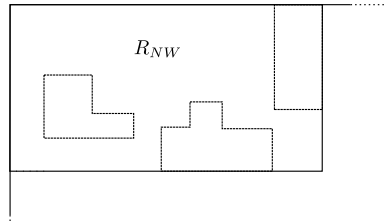

(f)

Fig. 23 Example of invasion of the dual rectangle $R_{1}$. Only the support of the relevant clusters are drawn and the parity of different clusters is not indicated. The set $\Lambda\left(R_{1}\right)$ contains a supercritical bridge belonging to cluster $A$ (Fig. 23(a)). Growing this bridge via the construction of its northern rectangle and its subsequent maximal expansion leads to the supercritical rectangle $R_{2}$ (Fig. 23(b)). Next, the invasion of $\Lambda\left(R_{2}\right)$ has to be performed in order to complete the invasion of $R_{1}$. The set $\Lambda\left(R_{2}\right)$ contains a supercritical bridge belonging to cluster $B$, which is grown into the supercritical rectangle $R_{3}$ (Fig. 23(c)). Note that $R_{3}$ partly covers the support of $\tilde{R}_{1}$ and that $R_{3}$ and $\bar{R}_{1}$ are adjacent. The invasion of $R_{2}$ proceeds via the invasion of $R_{3}$. Since $\Lambda\left(R_{3}\right)$ is empty, the invasion of $R_{3}$ is carried out by adding 12-bars to the left-side of $R_{3}$ until $\tilde{R}_{2}$ is at dual distance 1 . After that a maximal expansion produces a dual rectangle that covers the support of $\tilde{R}_{2}$ (Fig. 23(d)). The new dual rectangle $R_{2}$ is adjacent to $\bar{R}_{1}$. The two rectangles are merged and a maximal expansion gives a new rectangle $R_{1}$ (Fig. 23(e)). Now $\Lambda\left(R_{1}\right)$ is empty and can be filled by adding 12-bars to the left-side of $R_{1}$ until the rectangle $R_{N W}$ is obtained (Fig. 23(f))

of those sites whose vertical coordinates are $\geq b_{i}$ and whose horizontal coordinates are $>a_{i}$. If $R_{i}$ is on the right of $R_{i-1}$, then let $\left(a_{i}, b_{i}\right)$ denote, respectively, the horizontal and the vertical coordinate of the lower left-most particle (which is of type 1) of $R_{i}$, and call $\Lambda\left(R_{i}\right)$ the subset of $\Lambda\left(R_{i-1}\right)$ consisting of those sites whose vertical coordinates are $\geq b_{i}$ and whose horizontal coordinates are $<a_{i}$. In words, $\Lambda\left(R_{i}\right)$ consists of those sites of $\Lambda\left(R_{i-1}\right)$ between $R_{i-1}$ and $R_{i}$. Perform the cleaning mechanism and scan $\Lambda\left(R_{i}\right)$ in the lexicographic order. There are again several cases.

1. $\Lambda\left(R_{i}\right)$ is empty. Call $R^{\max }$ the rectangle with the largest vertical side between $R_{i}$ and $\bar{R}_{i-1}$ (in case of tie, without loss of generality choose $R^{\max }=R_{i}$ ) and call $R^{\text {min }}$ the other rectangle. Add vertical 12-bars on the side of $R^{\min }$ facing $R^{\max }$ until (depending on the parity of the rectangles) it becomes adjacent (different parity) to $R^{\max }$ or it is at distance 1 (same parity) from $R^{\max }$. In the first case, slide the extended $R^{\min }$ onto $R^{\max }$. Perform the maximal expansion of $R^{\max }$, and call $R_{i-1}$ the rectangle obtained in this way, whose support contains $\operatorname{supp}\left(R_{i}\right) \cup R_{i-1} \cup \Lambda\left(R_{i-1}\right)$. Restart the invasion of $R_{i-1}$.

2. The first horizontal bridge $b_{i+1}$ encountered in $\Lambda\left(R_{i}\right)$ has length $<\ell^{\star}$. Remove the particles of the (south)-support of the bridge, lowering the energy of the configuration, and restart the invasion of $R_{i}$.

3. The first horizontal bridge $b_{i+1}$ encountered in $\Lambda\left(R_{i}\right)$ has length $\geq \ell^{\star}$. First turn $b_{i+1}$ into the 12-bar $\bar{b}_{i+1}$, then build the 2-tiled rectangle $r_{i+1}=\sqcap\left(\bar{b}_{i+1}\right)$, after that expand $r_{i}$ to $R_{i+1}=\mathcal{R}^{\top}\left(r_{i+1}\right)$, and finally perform the cleaning mechanism. Call $\tilde{R}_{i}$ what is left of $R_{i}$ after the maximal expansion of $R_{i+1}$. The following cases are possible. 
(a) $R_{i+1}$ is not supercritical. This implies $\tilde{R}_{i}=R_{i}$. Remove $R_{i+1}$ from $\Lambda$, put $R_{i}=$ $\tilde{R}_{i}$, and restart the invasion of $R_{i}$.

(b) $R_{i+1}$ is supercritical and $\tilde{R}_{i}=\emptyset$. Change the name of $R_{i+1}$ to $R_{i}$, and restart the invasion of $R_{i}$.

(c) $R_{i+1}$ is supercritical and is adjacent to $\tilde{R}_{i}$. Note that both rectangles touch the north-side of $\Lambda^{-}$. Slide the rectangle with the shorter vertical length onto the other rectangle and perform again the maximal expansion of the rectangle that has not been moved during the sliding. These steps bring the configuration to a rectangle whose support contains $\operatorname{supp}\left(R_{i+1}\right) \cup \operatorname{supp}\left(R_{i}\right) \cup \Lambda\left(R_{i}\right)$. Call this rectangle $R_{i}$ and restart the invasion of $R_{i}$.

(d) $R_{i+1}$ is supercritical and is not adjacent to $\tilde{R}_{i}$. This implies $\tilde{R}_{i}=R_{i}$. Start the invasion of $R_{i+1}$.

The finiteness of $\Lambda$ ensures that the algorithm eventually terminates.

Open Access This article is distributed under the terms of the Creative Commons Attribution Noncommercial License which permits any noncommercial use, distribution, and reproduction in any medium, provided the original author(s) and source are credited.

\section{References}

1. Alonso, L., Cerf, R.: The three dimensional polyominoes of minimal area. Electron. J. Comb. 3 (1996). Research Paper 27

2. Bovier, A.: Metastability. In: Kotecký, R., (ed.) Methods of Contemporary Mathematical Statistical Physics. Lecture Notes in Mathematics, vol. 1970, pp. 177-221. Springer, Berlin (2009)

3. den Hollander, F., Nardi, F.R., Troiani, A.: Metastability for Kawasaki dynamics at low temperature with two types of particles. Electron. J. Probab. (submitted). arXiv:1101.6069v1

4. den Hollander, F., Nardi, F.R., Troiani, A.: Kawasaki dynamics with two types of particles: critical droplets. Manuscript in preparation

5. Manzo, F., Nardi, F.R., Olivieri, E., Scoppola, E.: On the essential features of metastability: tunnelling time and critical configurations. J. Stat. Phys. 115, 591-642 (2004) 